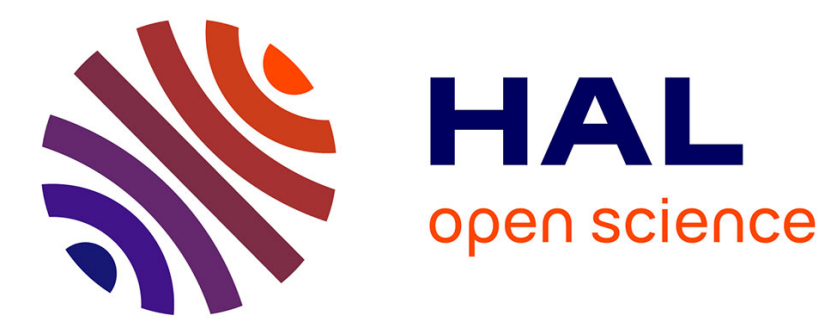

\title{
Acoustic inverse scattering using topological derivative of far-field measurements-based L2 cost functionals
}

\author{
Cédric Bellis, Marc Bonnet, Fioralba Cakoni
}

\section{To cite this version:}

Cédric Bellis, Marc Bonnet, Fioralba Cakoni. Acoustic inverse scattering using topological derivative of far-field measurements-based L2 cost functionals. Inverse Problems, 2013, pp.075012. 10.1088/0266$5611 / 29 / 7 / 075012$. hal-00835030

\section{HAL Id: hal-00835030 \\ https://hal.science/hal-00835030}

Submitted on 17 Jun 2013

HAL is a multi-disciplinary open access archive for the deposit and dissemination of scientific research documents, whether they are published or not. The documents may come from teaching and research institutions in France or abroad, or from public or private research centers.
L'archive ouverte pluridisciplinaire HAL, est destinée au dépôt et à la diffusion de documents scientifiques de niveau recherche, publiés ou non, émanant des établissements d'enseignement et de recherche français ou étrangers, des laboratoires publics ou privés. 


\title{
Acoustic inverse scattering using topological derivative of far-field measurements-based $L^{2}$ cost functionals
}

\author{
Cédric Bellis ${ }^{1}$, Marc Bonnet $^{2}$, Fioralba Cakoni $^{3}$ \\ ${ }^{1}$ Department of Applied Physics and Applied Mathematics, Columbia University, \\ New York, USA \\ ${ }^{2}$ POems (UMR 7231 CNRS-INRIA-ENSTA), ENSTA, Palaiseau, France \\ ${ }^{3}$ Department of Mathematical Sciences, University of Delaware, Newark, USA \\ E-mail: bellis@lma.cnrs-mrs.fr, mbonnet@ensta.fr, cakoni@math.udel.edu
}

\begin{abstract}
Originally formulated in the context of topology optimization, the concept of topological derivative has also proved effective as a qualitative inversion tool for wave-based identification of finite-sized objects. This approach remains however largely based on a heuristic interpretation of the topological derivative, whereas most other qualitative approaches to inverse scattering are backed by a mathematical justification. As an effort towards bridging this gap, this study focuses on a topological derivative approach applied to the $L^{2}$-norm of the misfit between far-field measurements. Either an inhomogeneous medium or a finite number of point-like scatterers are considered, using either the Born approximation or a full scattering model. Topological derivativebased imaging functionals are analyzed using a suitable factorization of the far-field operator, for each of the considered cases, in order to characterize their behavior and assess their ability to reconstruct the unknown scatterer(s). Results include the justification of the usual sign heuristic underpinning the method for (i) the Born approximation and (ii) full-scattering models limited to moderately strong scatterers. Semi-analytical and numerical examples are presented. Within the chosen framework, the topological derivative approach is finally discussed and compared to other wellknown qualitative methods.
\end{abstract}

Keywords: Topological derivative, inverse scattering, far-field measurements.

\section{Introduction}

Inverse scattering has been the subject of intense studies over the last twenty years, and has in particular spawned the growth and flourishing of qualitative, samplingbased, methods $[1,2,3]$ that aim at providing a robust and computationally effective alternative to more customary approaches based on successive linearizations or iterative optimization methods. Since the scattering operator (see e.g. [4]) plays a central role in forward scattering problems, inverse scattering methods have early been designed as strategies to extract the information contained in the corresponding measurement operator $[5,1]$. The following brief bibliographical review on the subject highlights the importance of this idea and connections between some of these methods: 
- Exploitation of the spectrum of the measurement or the scattering operator [6, 7, 8].

- Linear sampling and factorization methods, seen as two comparable strategies [9] to extract information from the scattering operator in a "simple" way $[2,10]$.

- Parallels between the MUSIC algorithm, linear sampling and factorization methods $[11,12,10]$.

- Use of the MUSIC algorithm to deal with inverse scattering problems [13, 14].

- MUSIC algorithm and time reversal [13, 15].

- Time reversal and imaging [16, 17, 18, 19].

- Exploitation of the spectrum of the time reversal operator and DORT method (French acronym for Decomposition of the Time-Reversal Operator) [20, 21, 22].

In addition to all the aforementioned approaches, the concept of topological derivative, which first appeared in the context of topological optimization of structures [23, 24], revolves around the quantification of the leading perturbation of a given cost functional, namely its topological derivative, due to the creation of a virtual object of vanishingly small characteristic size at a prescribed location $z$ inside the background (i.e. defectfree) medium. Over the last few years the topological derivative of data misfit cost functionals has been investigated in a variety of inverse scattering situations as a way to define an indicator function of the hidden objects, see e.g. [25, 26, 27, 28, 29, 30, 31, 32]. While defining and formulating the topological derivative of a given cost functional is mathematically rigorous, its subsequent use for imaging a given domain remains largely heuristic. Nonetheless, the method has been shown to lead to efficient and robust imaging functionals; moreover, it is very flexible in terms of exploitable data and misfit functionals, and easily implementable using classical computational methods [25, 26, 27, 28, 32]. On the other hand, investigations towards a better theoretical understanding of this approach have begun only recently. For example, [28] points out the analogy with time reversal, and the imaging of a single small scatterer is mathematically studied in [29], where proofs of stability with respect to medium or measurement noises are also given.

This article focuses on indicator functions provided by the topological derivative of $L^{2}$ misfit cost functionals, in the context of inverse scattering by an acoustic medium characterized by a inhomogeneous refraction index $n$. The available data is assumed to consist of measurements of the scattered far-field patterns, gathered into the farfield operator $F$. This work aims at providing a mathematical basis to the, until now heuristic, use of the topological derivative approach in this context. The behavior of the indicator function will be studied depending on the location of the sampling point $z$, the choice of the trial refraction index featured in the asymptotic analysis of the cost functional and the values of characteristic frequency, contrast $q=n^{2}-1$ and obstacle size. Scattering by either spatially extended inhomogeneities or a collection of pointlike scatterers will be considered, either under the weak-scatterer (Born) approximation or using a full scattering model taking into account multiple-scattering effects. In the latter case, a full justification of the topological derivative approach will be obtained only within limitations on the frequency and scatterer characteristics. The analysis, 
and the justification results obtained, exploits a fundamental relation, established here, between the topological derivative and the far-field operator $F$. In conjunction with the use of explicit factorizations of $F$ in the different situations considered, this relation is instrumental in gaining insight into the workings of the topological derivative approach and putting it in perspective within the general class of sampling methodologies for inverse scattering, which are the two main objectives of this work.

This article is accordingly organized as follows. Section 2 gathers background material on the forward scattering problem. Section 3 is then devoted to the topological derivative of far-field measurement-based least-squares cost functionals. Its validity as an indicator function is first justified under the Born approximation, and then partially extended to the full scattering model, mainly by using connections with the far-field operator and exploiting its known factorization. The section ends with analytical and numerical examples. Similar analyses are next carried out in Section 4 for the identification of spatially small, point-like obstacles (with full scattering modelled using the Foldy-Lax approximation), and again completed by numerical results. Finally, Section 5 puts the topological derivative approach in a broader perspective, by discussing both its specificities and its connections with other sampling methods.

\section{Forward acoustic scattering problem}

Consider a infinite homogeneous background acoustic medium, occupying all of $\mathbb{R}^{d}$ with $d=2$ or 3 and characterized by the constant wave velocity $c_{0}$. Let $D=\bigcup_{m=1}^{M} D_{m} \subset \mathbb{R}^{d}$ be a open and bounded domain with Lipschitz boundary $\partial D$ and such that $\mathbb{R}^{d} \backslash \bar{D}$ is connected. $D$ denotes the support of a scattering inhomogeneity characterized by a realvalued contrast function $q \in L^{\infty}(D)$, of constant sign in each connected component $D_{m}$, and for which there exists $n_{D}>0$ such that $1+q(x) \geq n_{D}^{2}$ for all $x \in D$. The contrast $q$ is related to the index of refraction $n=c / c_{0}$ (with $c$ denoting the wave velocity in $D$ ) by $q=n^{2}-1$. It is extended to $\mathbb{R}^{d}$ by setting $q=0$, i.e. $n=1$, in $\mathbb{R}^{3} \backslash \bar{D}$.

Let $k=\omega / c_{0}$ be the wave number in the background medium. Considering an incident field $u_{i}$ that is a known solution of the unperturbed Helmholtz equation $\Delta u+k^{2} u=0$ in $\mathbb{R}^{d}$, the forward acoustic scattering problem under consideration is

$$
\begin{array}{ll}
\Delta u+k^{2}(1+q) u=0 & \text { in } \mathbb{R}^{d}, \\
u=u_{i}+v, & \\
\frac{\partial v}{\partial|x|}-\mathrm{i} k v=\mathcal{O}\left(|x|^{-(d+1) / 2}\right) & \text { for }|x| \rightarrow \infty
\end{array}
$$

where $u \in H_{\mathrm{loc}}^{1}\left(\mathbb{R}^{d}\right)$ is the total acoustic field, the scattered field $v$ satisfies the Sommerfeld radiation condition (1c) uniformly in $\hat{x}=x /|x| \in \mathbb{S}$, with $\mathbb{S}$ denoting the unit circle if $d=2$ or the unit sphere if $d=3$. The latter condition implies the existence of a far-field pattern $v^{\infty}$ such that

$$
u(x)=u_{i}(x)+\gamma_{d} \frac{e^{\mathrm{i} k|x|}}{|x|^{(d-1) / 2}} v^{\infty}(\hat{x})+\mathcal{O}\left(|x|^{-(d+1) / 2}\right) \quad \text { for }|x| \rightarrow \infty,
$$


where the parameter $\gamma_{d}$ is such that

$$
\gamma_{2}=e^{\mathrm{i} \pi / 4} / \sqrt{8 \pi k}, \quad \gamma_{3}=1 / 4 \pi
$$

The radiating fundamental solution $\Phi$ of the Helmholtz equation in $\mathbb{R}^{d}$ is given by

$$
\Phi(x, y)=\frac{\mathrm{i}}{4} H_{0}^{(1)}(k|x-y|) \quad(\text { for } d=2), \quad \Phi(x, y)=\frac{e^{\mathrm{i} k|x-y|}}{4 \pi|x-y|} \quad(\text { for } d=3),
$$

where $H_{0}^{(1)}$ is the Hankel function of the first kind and order zero. Denoting by $h(\cdot, \theta)$ the plane wave propagating in the direction $\theta \in \mathbb{S}$ defined by

$$
h(x, \theta)=e^{\mathrm{i} k x \cdot \theta}, \quad x \in \mathbb{R}^{d},
$$

the far-field pattern $\Phi_{y}^{\infty}(\hat{x})$ of $\Phi$, such that the asymptotic expansion

$$
\Phi(x, y)=\gamma_{d} \frac{e^{\mathrm{i} k|x|}}{|x|^{(d-1) / 2}} \Phi_{y}^{\infty}(\hat{x})+\mathcal{O}\left(|x|^{-(d+1) / 2}\right) \quad \text { for }|x| \rightarrow \infty
$$

holds, is given by

$$
\Phi_{y}^{\infty}(\hat{x})=h(y,-\hat{x})=\overline{h(y, \hat{x})} .
$$

Solving problem $(1 \mathrm{a}-\mathrm{c})$ is known [5] to be equivalent to finding the solution $u \in L^{2}(D)$ of the Lippmann-Schwinger integral equation

$$
\left(I-S T_{b}\right) u=u_{i}
$$

where the volume potential operator $S: L^{2}(D) \rightarrow L^{2}(D)$ is defined by

$$
S \varphi(x):=\int_{D} \varphi(y) \Phi(x, y) \mathrm{d} V_{y}, \quad x \in D,
$$

$I$ is the identity, and the operator $T_{b}: L^{2}(D) \rightarrow L^{2}(D)$ is defined by $T_{b} \varphi=k^{2} q \varphi$. Then, the scattered field $v$ in $\mathbb{R}^{d} \backslash \bar{D}$ is given by the explicit integral representation

$$
v(x)=S T_{b} u(x), \quad x \in \mathbb{R}^{d} \backslash \bar{D},
$$

with $S$ denoting the $L^{2}(D) \rightarrow H_{\text {loc }}^{1}\left(\mathbb{R}^{d}\right)$ extension of the volume potential operator (7). In particular, using (2) and (4) in (8), the far-field pattern $v^{\infty}$ is then given by

$$
v^{\infty}(\hat{x})=\int_{D} k^{2} q(y) u(y) \overline{h(y, \hat{x})} \mathrm{d} V_{y}, \quad \hat{x} \in \mathbb{S} .
$$

The introduction of the parameter $\gamma_{d}$ in definitions (2) and (4) of the far-field patterns makes the ensuing analysis and results independent, to a large extent, of the spatial dimension $d$.

If the incident field is chosen as a plane wave propagating in the direction $\theta \in \mathbb{S}$, i.e. $u_{i}=h(\cdot, \theta)$, the corresponding far-field pattern $v^{\infty}$ is denoted $A(\cdot, \theta)$, i.e.:

$$
u(x)=h(x, \theta)+\gamma_{d} \frac{e^{\mathrm{i} k|x|}}{|x|^{(d-1) / 2}} A(\hat{x}, \theta)+\mathcal{O}\left(|x|^{-(d+1) / 2}\right) \quad \text { for }|x| \rightarrow \infty .
$$

Then, if $D$ is illuminated instead by a continuous superposition of plane waves, i.e. $u_{i}$ is a Herglotz wave with density $g \in L^{2}(\mathbb{S})$ :

$$
u_{i}(x)=\int_{\mathbb{S}} h(x, \theta) g(\theta) \mathrm{d} S_{\theta} \quad x \in \mathbb{R}^{d},
$$


the corresponding far-field pattern $v^{\infty}$ is expressed in terms of the far-field operator $F: L^{2}(\mathbb{S}) \rightarrow L^{2}(\mathbb{S})$ with kernel $A:$

$$
v^{\infty}(\hat{x})=F g(\hat{x}), \quad F g(\hat{x}):=\int_{\mathbb{S}} A(\hat{x}, \theta) g(\theta) \mathrm{d} S_{\theta},
$$

which is known [10] to be normal (i.e. $F F^{\star}=F^{\star} F$ ) since $q$ is real-valued. Finally, the Herglotz operator $H$ and its adjoint $H^{\star}$ are defined for later reference:

$$
\begin{aligned}
H: L^{2}(\mathbb{S}) \rightarrow L^{2}(D), H g(x)=\int_{\mathbb{S}} h(x, \theta) g(\theta) \mathrm{d} S_{\theta}, \\
H^{\star}: L^{2}(D) \rightarrow L^{2}(\mathbb{S}), H^{\star} \phi(\hat{x})=\int_{D} h(y,-\hat{x}) \phi(y) \mathrm{d} V_{y}=\int_{D} \overline{h(y, \hat{x})} \phi(y) \mathrm{d} V_{y} .
\end{aligned}
$$

The following identity satisfied by $H$ will later prove very useful:

Lemma 1. Let $\zeta_{0}$ denote the function defined for $x \in \mathbb{R}^{d}$ by

$$
\zeta_{0}(x)=2 \pi J_{0}(k|x|) \quad(d=2), \quad \zeta_{0}(x)=4 \pi j_{0}(k|x|)=4 \pi \frac{\sin (k|x|)}{k|x|} \quad(d=3),
$$

where $J_{0}$ is the Bessel function of the first kind and order zero and $j_{0}$ its spherical counterpart. Then, one has

$$
H \Phi_{z}^{\infty}(y)=\zeta_{0}(y-z), \quad y \in D
$$

Proof. By definition of $H$ and $\Phi_{z}^{\infty}$, one has that

$$
H \Phi_{z}^{\infty}(y)=\int_{\mathbb{S}} e^{\mathrm{i} k(y-z) \cdot \theta} \mathrm{d} S_{\theta}, \quad y \in D .
$$

The above integral is then readily seen to coincide (up to the appropriate constant factor) with the integral representation of the relevant Bessel function (see e.g. [33], formulae 10.9.1 and 10.54.1).

\section{Inverse scattering by an inhomogeneous medium}

\subsection{Topological derivative of $L^{2}$ cost functionals}

The illumination by an incident wave $u_{i}$ of a given trial obstacle $D_{\star}$, characterized by an assumed contrast $q_{\star}$ such that $1+q_{\star} \geq n_{\star}^{2}>0$ in $D_{\star}$ and $\bar{D}_{\star}=\operatorname{supp}\left(q_{\star}\right)$, generates the corresponding far-field pattern $v_{\star}^{\infty}$. Therefore, in order to quantify the discrepancy between $D_{\star}$ and the obstacle $D$ to be identified, one may introduce the following type of least-squares cost functional $\mathcal{J}$ evaluating the misfit between far-field measurements $v_{\text {obs }}^{\infty}$ of (9) and their trial counterpart $v_{\star}^{\infty}$ :

$$
\mathcal{J}\left(D_{\star}, q_{\star}\right):=\int_{\mathbb{S}} \frac{1}{2}\left|v_{\star}^{\infty}(\hat{x})-v_{\mathrm{obs}}^{\infty}(\hat{x})\right|^{2} \mathrm{~d} S_{\hat{x}}
$$

One further assumes that the data $v_{\text {obs }}$ featured in (15) consists of noise-free measurements on $\mathbb{S}$ of the acoustic field scattered by $D$, i.e. $v_{\text {obs }}^{\infty} \equiv v^{\infty}$. The above functional assumes data from just one incident wave; multiple data may then be taken into account via finite sums or continuous superposition of functionals, as required. 
Sampling methods are commonly investigated under the assumption that fullaperture far-field data be available for all possible directions of incident plane waves, i.e. that the kernel $A(\hat{x}, \theta)$ of $F$ be known from measurements for all $\hat{x}, \theta \in \mathbb{S}$, as this data uniquely determines the refraction index $q$ (see e.g. Theorem 6.26 in [1]). The cost functional of type (15) corresponding to this situation, denoted hereinafter as $\mathcal{J}_{\mathbb{S}}$, is defined by

$$
\mathcal{J}_{\mathbb{S}}\left(D_{\star}, q_{\star}\right):=\int_{\mathbb{S}} \int_{\mathbb{S}} \frac{1}{2}\left|A_{\star}(\hat{x}, \theta)-A(\hat{x}, \theta)\right|^{2} \mathrm{~d} S_{\hat{x}} \mathrm{~d} S_{\theta},
$$

The case of a single incident wave of Herglotz type, i.e. of the form $u_{i}=H g$ for some $g \in L^{2}(\mathbb{S})$, is also of interest, especially when $g$ is selected on the basis of the full experimental information $A(\hat{x}, \theta)$. The corresponding cost functional of type (15), denoted by $\mathcal{J}[g]$ to emphasize its dependence on the Herglotz density $g$, is defined by

$$
\mathcal{J}[g]\left(D_{\star}, q_{\star}\right):=\int_{\mathbb{S}} \frac{1}{2}\left|F g(\hat{x})-F_{\star} g(\hat{x})\right|^{2} \mathrm{~d} S_{\hat{x}} .
$$

The remainder of this article is mainly focused on studying the topological derivative of the cost functionals $\mathcal{J}_{\mathbb{S}}$ and $\mathcal{J}[g]$ as means for the qualitative reconstruction of $D$.

Topological derivative. For a given sampling point $z \in \mathbb{R}^{d}$, let the trial obstacle be endowed with a uniform contrast $q_{\star}$ and geometrically defined by $D_{\star}=D_{z}^{\varepsilon}:=z+\varepsilon \mathcal{D}$, where $\mathcal{D} \subset \mathbb{R}^{d}$ is a fixed open set containing the origin and with volume measure $|\mathcal{D}|$. The topological derivative $\mathcal{T}(z)$ of $\mathcal{J}$ at $z$ is defined through the asymptotic expansion of $\mathcal{J}$ as $\varepsilon \rightarrow 0$ :

$$
\mathcal{J}\left(D_{z}^{\varepsilon}, q_{\star}\right) \underset{\varepsilon \rightarrow 0}{=} \mathcal{J}(\emptyset)+\eta(\varepsilon) \mathcal{T}(z)+o\left(\left\|v_{\varepsilon, z}^{\infty}\right\|_{L^{2}(\mathbb{S})}\right)
$$

where $v_{\varepsilon, z}^{\infty}$ is the far-field pattern arising from the scattering of $u_{i}$ by $D_{z}^{\varepsilon}, \eta(\varepsilon)$ defines the leading asymptotic behavior of $\mathcal{J}$ as $\varepsilon \rightarrow 0$ and $\mathcal{J}(\emptyset)$ is the value of $\mathcal{J}$ in the absence of any trial obstacle. Now, using the first-order Taylor expansion of $\mathcal{J}\left(D_{z}^{\varepsilon}, q_{\star}\right)$ with respect to $v_{\varepsilon, z}^{\infty}, \mathcal{T}(z)$ and $\eta(\varepsilon)$ are determined by identification in the asymptotic equality (see e.g. [30])

$$
\eta(\varepsilon) \mathcal{T}(z) \underset{\varepsilon \rightarrow 0}{\sim}-\operatorname{Re}\left[\int_{\mathbb{S}} \overline{v^{\infty}(\hat{x})} v_{\varepsilon, z}^{\infty}(\hat{x}) \mathrm{d} S_{\hat{x}}\right] .
$$

The scattered field for the infinitesimal inclusion $D_{z}^{\varepsilon}$ is known [34] to have the behavior

$$
v_{\varepsilon, z}(x)=\varepsilon^{d}|\mathcal{D}| k^{2} q_{\star} u_{i}(z) \Phi(x, z)+o\left(\varepsilon^{d}\right),
$$

at any point $x \neq z$, implying that the corresponding far-field pattern reads

$$
v_{\varepsilon, z}^{\infty}(\hat{x})=\varepsilon^{d}|\mathcal{D}| k^{2} q_{\star} u_{i}(z) h(z,-\hat{x})+o\left(\varepsilon^{d}\right) .
$$

Considering an incident wave $u_{i}=H g$ in $(21)$ for some $g \in L^{2}(\mathbb{S})$, one therefore has $v_{\varepsilon, z}^{\infty}(\hat{x})=F_{z}^{\varepsilon} g(\hat{x})$, with the far-field operator $F_{z}^{\varepsilon}$ having the expansion

$$
F_{z}^{\varepsilon} g(\hat{x})=\varepsilon^{d} F_{z}^{0} g(\hat{x})+o\left(\varepsilon^{d}\right) .
$$

The operator $F_{z}^{0}$ therefore has (in the sense of definition (12)) a kernel $A_{z}^{0}$, given by

$$
A_{z}^{0}(\hat{x}, \theta)=|\mathcal{D}| k^{2} q_{\star} h(z, \theta) h(z,-\hat{x})=|\mathcal{D}| k^{2} q_{\star} \overline{\Phi_{z}^{\infty}(\theta)} \Phi_{z}^{\infty}(\hat{x})
$$


Replacing $v^{\infty}(\hat{x})$ by $F g(\hat{x})$ and $v_{\varepsilon, z}^{\infty}(\hat{x})$ by $\varepsilon^{d} F_{z}^{0} g(\hat{x})+o\left(\varepsilon^{d}\right)$ in (19), the asymptotic behavior $\eta(\varepsilon)$ and the topological derivative $\mathcal{T}[g]$ of $\mathcal{J}[g]$ are found to be $\eta(\varepsilon)=\varepsilon^{d}$ and

$$
\mathcal{T}[g](z)=-\operatorname{Re}\left[\int_{\mathbb{S}} \overline{F g(\hat{x})} F_{z}^{0} g(\hat{x}) \mathrm{d} S_{\hat{x}}\right] .
$$

Similarly, the same behaviour $\eta(\varepsilon)=\varepsilon^{d}$ is found for $\mathcal{J}_{\mathbb{S}}$, with its topological derivative $\mathcal{T}_{\mathbb{S}}(z)$ obtained as

$$
\mathcal{T}_{\mathbb{S}}(z)=-\operatorname{Re}\left[\int_{\mathbb{S}} \int_{\mathbb{S}} \overline{A(\hat{x}, \theta)} A_{z}^{0}(\hat{x}, \theta) \mathrm{d} S_{\hat{x}} \mathrm{~d} S_{\theta}\right]
$$

by replacing $v^{\infty}(\hat{x})$ by $A(\hat{x}, \theta)$ and $v_{\varepsilon, z}^{\infty}(\hat{x})$ by $\varepsilon^{d} A_{z}^{0} g(\hat{x}, \theta)+o\left(\varepsilon^{d}\right)$ in (19), retaining the leading contribution as $\varepsilon \rightarrow 0$ and integrating the result over $\theta \in \mathbb{S}$. In addition, key relationships hold between topological derivatives and the far-field operator:

Proposition 1. The topological derivatives (24) and (25) can be recast as follows in terms of the far-field operator $F$ associated with the unknown scatterer $(D, q)$ :

$$
\begin{aligned}
\mathcal{T}[g](z) & =-|\mathcal{D}| k^{2} q_{\star} \operatorname{Re}\left[\left(g, \Phi_{z}^{\infty}\right)_{L^{2}(\mathbb{S})}\left(\Phi_{z}^{\infty}, F g\right)_{L^{2}(\mathbb{S})}\right], \\
\mathcal{T}_{\mathbb{S}}(z) & =-|\mathcal{D}| k^{2} q_{\star} \operatorname{Re}\left[\left(\Phi_{z}^{\infty}, F \Phi_{z}^{\infty}\right)_{L^{2}(\mathbb{S})}\right]
\end{aligned}
$$

Proof. Formula (26a) results from using $F_{z}^{0} g(\hat{x})=|\mathcal{D}| k^{2} q_{\star}\left(g, \Phi_{z}^{\infty}\right)_{L^{2}(\mathbb{S})} \Phi_{z}^{\infty}(\hat{x})$ (by virtue of (23)) in (24) and treating the resulting integral over $\mathbb{S}$ as a scalar product in $L^{2}(\mathbb{S})$.

To establish (26b), one first uses definitions (23) of $A_{z}^{0}$ and invokes the reciprocity property $A(\hat{x}, \theta)=A(-\theta,-\hat{x})$ of $F([5]$, Theorem 8.8), to obtain

$$
\begin{aligned}
\overline{A(\hat{x}, \theta)} A_{z}^{0}\left(\hat{x}, \theta^{\prime}\right) & =-|\mathcal{D}| k^{2} q_{\star} \overline{A(-\theta,-\hat{x}) \overline{h(z,-\hat{x})}} h\left(z, \theta^{\prime}\right) \\
& =-|\mathcal{D}| k^{2} q_{\star} \overline{A(-\theta,-\hat{x}) \Phi_{z}^{\infty}(-\hat{x})} \Phi_{z}^{\infty}\left(-\theta^{\prime}\right),
\end{aligned}
$$

for arbitrary $\theta, \theta^{\prime} \in \mathbb{S}$. Equation (26b) then follows from integrating the result over $\left(\theta, \theta^{\prime}\right) \in \mathbb{S} \times \mathbb{S}$.

Remark 1. The leading asymptotics of the least-squares cost functionals considered in this study is remarkably expressed, as in (24) or (25), in terms of the conjugated (i.e. time-reversed in the time domain) counterpart of the far-field pattern scattered by the unknown obstacle D. This observation directly leads to the key relations of Proposition 1 , which show the link between the topological derivative and the far-field operator.

\subsection{Sign heuristic}

The value $\mathcal{T}(z)$ quantifies the sensitivity of the featured cost functional $\mathcal{J}$ to the perturbation of the reference medium induced by the nucleation at $z \in \mathbb{R}^{d}$ of an infinitesimal obstacle with contrast $q_{\star}$. It is then natural to consider $z \mapsto \mathcal{T}(z)$ as a potential obstacle indicator function, as was previously done on several occasions (see $[25,26,27]$ and the references therein). The heuristic underlying this usage is as follows: if $q_{\star}$ is of the same sign than $q$, then the sought object $D$ (or the set thereof) is expected to be located at the sampling points $z$ at which $\mathcal{T}$ attains its most pronounced negative values, i.e. at which the introduction of a sufficiently small scatterer with a contrast of 
the same sign than that of $D$ induces the most pronounced decrease of $\mathcal{J}$. Note that no smallness requirement for $D$ is made in this approach, which is referred to hereinafter as the sign heuristic of the topological derivative. Up to now, this sign heuristic lacks rigorous justification but is supported by many numerical experiments. This study aims at investigating the validity of such heuristic and determining conditions under which it has a mathematical justification, in the limited framework of the identification of obstacles characterized by refraction index perturbations using far-field data.

\subsection{Born approximation}

It is natural to start by evaluating the validity of the topological derivative approach under the assumption of a weak scatterer approximation for the sought object $D$ before considering the more complex case of the full scattering model (Sec. 3.4). With reference to the Lippmann-Schwinger equation (6), this corresponds to situations where $k,|D|, q$ are such that $\left\|S T_{b}\right\| \ll 1$. If $\left\|S T_{b}\right\|<1$, equation (6) can be solved by fixed-point iterations. The first iterate, defined by $u_{b}=u_{i}$ in $D$ and $v_{b}=S T_{b} u_{i}$ in $\mathbb{R}^{d} \backslash \bar{D}$, constitutes the Born approximation. The Born approximation is indicated by the subscript or superscript " $b$ " affixed to all relevant fields and operators. Moreover, one notes that, in view of (20), the probing infinitesimal trial obstacle also obeys the Born approximation.

Under the weak scatterer approximation, the far-field operator has a known, and simple, factorization:

Lemma 2 ([10], Sec. 4.3). The far-field operator under the Born approximation, denoted by $F_{b}$, is defined by (12) with the kernel $A_{b}$ :

$$
A_{b}(\hat{x}, \theta)=\int_{D} k^{2} q(y) \overline{h(y, \hat{x})} h(y, \theta) d V_{y}, \quad \hat{x}, \theta \in \mathbb{S} .
$$

$F_{b}$ is compact and (for real-valued contrast $q \in L^{\infty}(D)$ ) self-adjoint; as such, it has a complete orthonormal system with eigenvalues $\lambda_{\ell}^{b} \in \mathbb{R}$ and eigenfunctions $\Psi_{\ell}^{b} \in L^{2}(\mathbb{S})$. Moreover, it admits the factorization

$$
F_{b}=H^{\star} T_{b} H
$$

where the operator $T_{b}: L^{2}(D) \rightarrow L^{2}(D)$ is defined by $T_{b} f=k^{2} q f$ and with $H, H^{\star}$ as defined by (13a) and (13b).

Applying this factorization to $(26 a, b)$ and using Lemma 1 for every occurrence of $H \Phi_{z}^{\infty}$, one obtains more explicit expressions for the topological derivatives:

Proposition 2. Under the Born approximation, the topological derivatives $\mathcal{T}^{b}[g]$ and $\mathcal{T}_{\mathbb{S}}^{b}$ are given (with the function $\zeta_{0}$ as defined in Lemma 1) by

$$
\begin{aligned}
\mathcal{T}^{b}[g](z) & =-|\mathcal{D}| k^{2} q_{\star} \operatorname{Re}\left[\left(\Phi_{z}^{\infty}, g\right)_{L^{2}(\mathbb{S})}\left(T_{b} H g, H \Phi_{z}^{\infty}\right)_{L^{2}(D)}\right] \\
& =-|\mathcal{D}| k^{4} q_{\star} \operatorname{Re}\left[\left(\Phi_{z}^{\infty}, g\right)_{L^{2}(\mathbb{S})} \int_{D} q(y) \zeta_{0}(y-z) H g(y) d V_{y}\right] \\
\mathcal{T}_{\mathbb{S}}^{b}(z) & =-|\mathcal{D}| k^{2} q_{\star}\left(H \Phi_{z}^{\infty}, T_{b} H \Phi_{z}^{\infty}\right)_{L^{2}(D)} \\
& =-|\mathcal{D}| k^{4} q_{\star} \int_{D} q(y) \zeta_{0}^{2}(y-z) d V_{y}
\end{aligned}
$$


where $g \in L^{2}(\mathbb{S})$ is arbitrary in (27a). Moreover, letting $g=\Psi_{k}^{b}$, where $\Psi_{k}^{b} \in L^{2}(\mathbb{S})$ is an eigenfunction of $F_{b}$ for the eigenvalue $\lambda_{k}^{b} \in \mathbb{R}, \lambda_{k}^{b} \neq 0$, so that $F_{b} g=\lambda_{k}^{b}$ g (see Lemma 2), one has

$$
\begin{aligned}
\mathcal{T}^{b}\left[\Psi_{k}^{b}\right](z) & =-|\mathcal{D}| k^{2} q_{\star} \lambda_{k}^{b}\left|\left(\Psi_{k}^{b}, \Phi_{z}^{\infty}\right)_{L^{2}(\mathbb{S})}\right|^{2} \\
& =-|\mathcal{D}| k^{2} q_{\star}\left(\lambda_{k}^{b}\right)^{-1}\left|\left(T_{b} H \Psi_{k}^{b}, H \Phi_{z}^{\infty}\right)_{L^{2}(D)}\right|^{2} .
\end{aligned}
$$

Proof. Formulae (27) are readily found by applying the factorization $F_{b}=H^{\star} T_{b} H$ to $(26 \mathrm{a}, \mathrm{b})$ and using Lemma 1 for every occurrence of $H \Phi_{z}^{\infty}$. Next, (26a) with $g=\Psi_{k}^{b}$ reads

$$
\mathcal{T}^{b}[g](z)=-|\mathcal{D}| k^{2} q_{\star} \operatorname{Re}\left[\left(\Phi_{z}^{\infty}, \Psi_{k}^{b}\right)_{L^{2}(\mathbb{S})}\left(F_{b} \Psi_{k}^{b}, \Phi_{z}^{\infty}\right)_{L^{2}(\mathbb{S})}\right] .
$$

Formula (28a) then results from setting $F_{b} \Psi_{k}^{b}=\lambda_{k}^{b} \Psi_{k}^{b}$ in the second inner product, whereas formula (28b) is obtained by setting $\Psi_{k}^{b}=\left(\lambda_{k}^{b}\right)^{-1} F_{b} \Psi_{k}^{b}$ in the first inner product and using the factorization $F_{b}=H^{\star} T_{b} H$.

Decay properties of the topological derivative. The topological derivatives as given in Proposition 2 involve the function $\zeta_{0}$ defined by (14), which has the well-known decay properties (see e.g. equations 10.7.8 and 10.52.3 in [33])

$$
\zeta_{0}(x)=\mathcal{O}\left(|x|^{-1 / 2}\right) \quad(\text { if } d=2), \quad \zeta_{0}(x)=\mathcal{O}\left(|x|^{-1}\right) \quad(\text { if } d=3), \quad|x| \rightarrow+\infty .
$$

As a result, $\mathcal{T}^{b}[g](z)$ and $\mathcal{T}_{\mathbb{S}}^{b}(z)$ decay away from $D$, as $\operatorname{dist}(z, D) \rightarrow \infty$, according to:

Proposition 3. The topological derivatives $\mathcal{T}^{b}[g]\left(\right.$ for any $g \in L^{2}(\mathbb{S})$ ) and $\mathcal{T}_{\mathbb{S}}^{b}$ have the following asymptotic behavior away from $D$ :

$$
\left|\mathcal{T}^{b}[g](z)\right|=\mathcal{O}\left(\operatorname{dist}(z, D)^{(1-d) / 2}\right), \quad\left|\mathcal{T}_{\mathbb{S}}^{b}(z)\right|=\mathcal{O}\left(\operatorname{dist}(z, D)^{1-d}\right), \quad|z| \rightarrow \infty .
$$

Moreover, the above estimate for $\mathcal{T}^{b}[g]$ can be refined in two cases: (i) for any density $g \in C^{0}(\mathbb{S})$, one has

$$
\left|\mathcal{T}^{b}[g](z)\right|=\mathcal{O}\left(|z|^{(1-d) / 2} \operatorname{dist}(z, D)^{(1-d) / 2}\right) \quad|z| \rightarrow \infty,
$$

and (ii) letting $g=\Psi_{k}^{b}$, where $\Psi_{k}^{b} \in L^{2}(\mathbb{S})$ is an eigenfunction of $F_{b}$ for the eigenvalue $\lambda_{k}^{b} \in \mathbb{R}$, one has

$$
\left|\mathcal{T}^{b}[g](z)\right|=\mathcal{O}\left(\operatorname{dist}(z, D)^{1-d}\right) \quad|z| \rightarrow \infty .
$$

Proof. Estimates (30) and (32) stem directly from invoking the Cauchy-Schwarz inequality and using (29) in (27a,b) and (28b), respectively. Moreover, estimate (31) follows from

$$
\left(\Phi_{z}^{\infty}, g\right)_{L^{2}(\mathbb{S})}=\int_{\mathbb{S}} e^{-\mathrm{i} k z \cdot \hat{x}} \overline{g(\hat{x})} \mathrm{d} S_{\hat{x}}=\mathcal{O}\left(|z|^{(1-d) / 2}\right),
$$

which holds for any $g \in C^{0}(\mathbb{S})$ by virtue of known properties of oscillatory integrals (see e.g. [35], Sec. 8.1).

The decay properties given by Proposition 3 show that $z \mapsto\left|\mathcal{T}^{b}[g](z)\right|$ and $z \mapsto\left|\mathcal{T}_{\mathbb{S}}^{b}(z)\right|$ already permit a qualitative identification of $D$. The sign heuristic usually underlying TD-based scatterer identification, which plays no role in Proposition 3, is now studied. 
Sign properties of the topological derivative. First, in the case where $q$ has a constant sign in $D$, it is clear from (27b) that

$$
\operatorname{sign}\left(\mathcal{T}_{\mathbb{S}}^{b}(z)\right)=-\operatorname{sign}\left(q_{\star} q\right)
$$

The topological derivative $\mathcal{T}_{\mathbb{S}}^{b}$, which is based on enough information for $(D, q)$ to be exactly identifiable, is thus found to have both desired attributes of TD-based imaging, namely (i) the sharpest decay (among the variants considered) away from $D$, and (ii) a sign which is consistent with its heuristic meaning $\left(\mathcal{J}_{\mathbb{S}}\right.$ decreases when a small trial scatterer $\operatorname{such}$ that $\operatorname{sign}\left(q_{\star}\right)=\operatorname{sign}(q)$ appears at $\left.z\right)$.

It does not appear that the sign of $\mathcal{T}^{b}[g]$ can be ascertained for arbitrary choices of $g$. However, for any eigenfunction $\Psi_{k}^{b} \in L^{2}(\mathbb{S})$ of $F_{b}$, one has $\operatorname{sign}\left(\lambda_{k}^{b}\right)=\operatorname{sign}(q)$ if $\operatorname{sign}(q)$ is constant in $D$ due to $F_{b}=H^{\star} T_{b} H$ and the definition of $T_{b}$. Hence, if $g=\Psi_{k}^{b}$, the topological derivative $\mathcal{T}^{b}\left[\Psi_{k}^{b}\right]$, which exploits one single combination of the available measurement, has characteristics similar to $\mathcal{T}_{\mathbb{S}}^{b}$, namely is such that

$$
\operatorname{sign}\left(\mathcal{T}^{b}\left[\Psi_{k}^{b}\right](z)\right)=-\operatorname{sign}\left(q_{\star} \lambda_{k}^{b}\right), \quad\left|\mathcal{T}^{b}\left[\Psi_{k}^{b}\right](z)\right|=\mathcal{O}\left(\operatorname{dist}(z, D)^{1-d}\right) \quad(|z| \rightarrow \infty)
$$

Now, the more complex case where $D$ is multiply connected (i.e. $\operatorname{supp}(q)=D=$ $\left.\cup_{m=1}^{M} D_{m}\right)$ with $q$ having constant sign in each connected component $D_{m}$, is considered. The topological derivative $\mathcal{T}_{\mathbb{S}}^{b}$ then satisfies the following corollary of Propositions 2, 3:

Corollary 1. Considering the case $d=3$, let $\sigma_{m}:=\operatorname{sign}\left(\left.q\right|_{D_{m}}\right), \sigma_{\star}:=\operatorname{sign}\left(q_{\star}\right)$, $\alpha:=16 \pi^{2} k^{2}|\mathcal{D}|$ and define

$$
Q_{m}:=\int_{D_{m}} q(y) d V_{y}, \quad \mathcal{I}_{m}(z):=-|\mathcal{D}| k^{4} \int_{D_{m}} q_{\star} q(y) \zeta_{0}^{2}(z-y) d V_{y} \quad(1 \leq m \leq M),
$$

noting that $\operatorname{sign}\left(\mathcal{I}_{m}(z)\right)=-\sigma_{\star} \sigma_{m}$. Then, for any exterior sampling point $z_{e} \notin D$, one has

$$
-\alpha \sum_{\sigma_{\star} \sigma_{m}=1} \frac{q_{\star} Q_{m}}{\operatorname{dist}\left(z, D_{m}\right)^{2}} \leq \mathcal{T}_{\mathbb{S}}^{b}\left(z_{e}\right) \leq-\alpha \sum_{\sigma_{\star} \sigma_{m}=-1} \frac{q_{\star} Q_{m}}{\operatorname{dist}\left(z, D_{m}\right)^{2}}
$$

and for any interior point $z_{i} \in D_{m_{0}} \subset D$, where $m_{0} \in\{1, \ldots, M\}$, one has

$$
\mathcal{I}_{m_{0}}\left(z_{i}\right)-\alpha \sum_{\substack{m \neq m_{0} \\ \sigma_{\star} \sigma_{m}=1}} \frac{q_{\star} Q_{m}}{\operatorname{dist}\left(z, D_{m}\right)^{2}} \leq \mathcal{T}_{\mathbb{S}}^{b}\left(z_{i}\right) \leq \mathcal{I}_{m_{0}}\left(z_{i}\right)-\alpha \sum_{\substack{m \neq m_{0} \\ \sigma_{\star} \sigma_{m}=-1}} \frac{q_{\star} Q_{m}}{\operatorname{dist}\left(z, D_{m}\right)^{2}}
$$

Proof. Inserting the definition (14) of $\zeta_{0}$ in (27b) and distinguishing between components $D_{m}$ where $q_{\star} q_{m}$ is positive or negative, one has

$$
\begin{aligned}
\mathcal{T}_{\mathbb{S}}^{b}(z)=\alpha\left\{-\sum_{\sigma_{\star} \sigma_{m}=1} \int_{D_{m}}\left|q_{\star} q(y)\right| \frac{\sin ^{2}(k|y-z|)}{|y-z|^{2}} \mathrm{~d} V_{y}\right. \\
\left.\quad+\sum_{\sigma_{\star} \sigma_{m}=-1} \int_{D_{m}}\left|q_{\star} q(y)\right| \frac{\sin ^{2}(k|y-z|)}{|y-z|^{2}} \mathrm{~d} V_{y}\right\}:=\alpha\left(-S^{+}+S^{-}\right) .
\end{aligned}
$$


If $z \notin D$, then for each $m=1, \ldots, M$

$$
0 \leq \int_{D_{m}}\left|q_{\star} q(y)\right| \frac{\sin ^{2}(k|y-z|)}{|y-z|^{2}} \mathrm{~d} V_{y} \leq \frac{\left|q_{\star} Q_{m}\right|}{\operatorname{dist}\left(z, D_{m}\right)^{2}}
$$

and (35) follows from applying this inequality to derive separate upper bounds of the positive sums $S^{-}$and $S^{+}$. Note that the upper and lower bounds of this inequality are respectively positive and negative.

If $z \in D$, then there exists $m_{0} \in\{1, \ldots, M\}$ such that $z \in D_{m_{0}}$ and one similarly obtains

$$
\mathcal{I}_{m_{0}}(z)-\alpha \sum_{\substack{m \neq m_{0} \\ \sigma \star \sigma m=1}} \frac{q_{\star} Q_{m}}{\operatorname{dist}\left(z, D_{m}\right)^{2}} \leq \mathcal{T}_{\mathbb{S}}^{b}(z) \leq \mathcal{I}_{m_{0}}(z)-\alpha \sum_{\substack{m \neq m_{0} \\ \sigma \star \sigma m=-1}} \frac{q_{\star} Q_{m}}{\operatorname{dist}\left(z, D_{m}\right)^{2}} .
$$

Remark 2. Proposition 3 and Corollary 1 give a key justification to the heuristic of the topological derivative approach presented in Section 3.1 under the Born approximation. Away from the scattering obstacle $D$, the expected decay of $\mathcal{T}_{\mathbb{S}}^{b}$ is $\mathcal{O}\left(\operatorname{dist}(z, D)^{1-d}\right)$. Moreover, for a given $m_{0}$, if the probing scatterer $D_{\star}$ is qualitatively of same nature than $D_{m_{0}}$ (i.e. if $\sigma_{\star} \sigma_{m_{0}}=1$ ), then $\mathcal{T}_{\mathbb{S}}^{b}$ exhibits large negative values inside $D_{m_{0}}$ provided that the effects of the remaining obstacle components $D_{m}, m \neq m_{0}$ can be neglected. On the contrary, if $D_{\star}$ and a given $D_{m_{0}}$ have opposite behaviors (i.e. $\sigma_{\star} \sigma_{m_{0}}=-1$ ), then pronounced positive values of $\mathcal{T}_{\mathbb{S}}^{b}$ occur inside $D_{m_{0}}$. This statement (that we do not formalize) is valid in the situations where the different geometrical components $D_{m}$ are sufficiently far from each other or when their material contrasts are relatively low.

The inequalities (36) show that there exist configurations where the reconstruction of a given $D_{m_{0}}$ can be skewed by the effects of the surrounding inhomogeneities, for example in terms of the sign of the topological derivative.

Remark 3. Corollary 1 does not have a simple counterpart for $d=2$ because $J_{0}^{2}(x) \leq$ $C x^{-1}$ only in the limit $x \rightarrow \infty$, whereas $j_{0}^{2}(x) \leq C x^{-2}$ for any $x>0$.

Topological derivative in convolutional form. Let $f \star g$ denote the convolution product of functions $f, g \in L^{2}\left(\mathbb{R}^{d}\right)$, i.e.

$$
[f \star g](x)=\int_{\mathbb{R}^{d}} f(y) g(x-y) \mathrm{d} V_{y} .
$$

By initial assumption, $q \in L^{\infty}(D)$ and has compact support $\bar{D}$; hence $q \in L^{2}\left(\mathbb{R}^{d}\right)$. The following proposition then follows by treating (27b) as a convolution integral:

Proposition 4. Let the function $\chi$ be defined by $\chi(x)=\zeta_{0}^{2}(x)$ for all $x \in \mathbb{R}^{d}$, with $\zeta_{0}$ as in Lemma 1. The topological derivative $\mathcal{T}_{\mathbb{S}}^{b}$ is then given by

$$
\mathcal{T}_{\mathbb{S}}^{b}(z)=-|\mathcal{D}| k^{4} q_{\star}[q \star \chi](z) .
$$

In formulation (37) of $\mathcal{T}_{\mathbb{S}}^{b}$, the convolution with the function $\chi$ acts as a filter on the material contrast function $q$, which has compact support. Therefore, the image provided by $\mathcal{T}_{\mathbb{S}}^{b}$ is expected to be a smoothed version of the actual object $(D, q)$, with the value $\mathcal{T}_{\mathbb{S}}^{b}(z)$ at a given sampling point $z$ related to the average of $q$ over a neighborhood of $z$. This idea of geometrical filtering is analyzed next. 
Remark 4. It is possible to find an asymptotic form of the right-hand side of (37) as $k \rightarrow \infty$. Within this type of approximation and owing to the known asymptotic behavior of $\zeta_{0}(z-y)$, the indicator function $\mathcal{T}_{\mathbb{S}}^{b}$ is expected to provide a sharper image of the sought obstacle. However, the relevance of this asymptotics remains constrained by the validity of the Born approximation (see discussion in Sec. 5.3). In particular, in the short-wavelength regime, the contrast function $q$ is restricted by (74) to very small values, which makes this type of approximation of very limited practical interest.

In order to obtain further insight on $\mathcal{T}_{\mathbb{S}}^{b}$ by exploiting its convolutional form (37), one introduces the Fourier transform of a function $f$ as $\hat{f}$ defined by

$$
\hat{f}(\xi)=\mathcal{F}[f](\xi)=\int_{\mathbb{R}^{d}} f(x) e^{-2 \pi \mathrm{i} x \cdot \xi} \mathrm{d} V_{x}
$$

The Fourier transform $\hat{\chi}$ of the radial function $\chi$ is also radial (see Theorem IV 3.3 in [36]), and simple calculations with the recourse to [37] show that

$$
\hat{\chi}(\xi)=\frac{4 \pi}{|\xi|} \frac{1}{\left(k^{2}-\pi^{2}|\xi|^{2}\right)^{1 / 2}} \quad\left(\text { if }|\xi|<\frac{k}{\pi}\right), \quad \hat{\chi}(\xi)=0 \quad\left(\text { if }|\xi|>\frac{k}{\pi}\right)
$$

for $d=2$, and

$$
\hat{\chi}(\xi)=\frac{4 \pi^{3}}{|\xi| k^{2}} \quad\left(\text { if }|\xi|<\frac{k}{\pi}\right), \quad \hat{\chi}(\xi)=0 \quad\left(\text { if }|\xi|>\frac{k}{\pi}\right)
$$

for $d=3$. From the identity (37), one obtains

$$
\mathcal{T}_{\mathbb{S}}^{b}(z)=-|\mathcal{D}| k^{4} q_{\star}[q \star \chi](z)=-|\mathcal{D}| k^{4} q_{\star} \mathcal{F}^{-1}[\hat{q}(\xi) \hat{\chi}(\xi)](z) .
$$

Since $\hat{\chi}(\xi)=0$ for $|\xi|>k / \pi$ for $d=2$ or 3 , equation (38) implies that spatial variations of $q$ within a characteristic length scale smaller than $\lambda / 2$, with $\lambda=2 \pi / k$, cannot be recovered. Hence, geometrical details of $D$ on a scale smaller than the resolution limit $\lambda / 2$ are filtered out in the reconstruction by the indicator function $\mathcal{T}_{\mathbb{S}}^{b}$.

In view of this resolution limit, it is natural to seek the transformation which, through deconvolution, will lead to the optimal reconstruction, in the $L^{2}$-norm sense, of the function $q$ from $\mathcal{T}_{\mathbb{S}}^{b}$. To do so, let the functions $\Theta$ and $\Pi$ be defined as follows in terms of their Fourier transforms:

$$
\begin{aligned}
& \hat{\Theta}(\xi)=1 / \hat{\chi}(\xi) \quad(\text { if } 0<|\xi|<k / \pi), \quad \hat{\Theta}(\xi)=0 \quad(\text { if }|\xi|>k / \pi), \\
& \hat{\Pi}(\xi)=1 \quad \text { (if }|\xi|<k / \pi), \quad \hat{\Pi}(\xi)=0 \quad \text { (if }|\xi|>k / \pi) \text {. }
\end{aligned}
$$

Using Theorem IV.3.3 in [36], duality properties of the Fourier transform and Eq. 19.1.3 in [37], the function $\Pi$ can be shown to be given by

$$
\Pi(x)=\frac{k}{\pi} \frac{J_{1}(2 k|x|)}{|x|} \quad(\text { if } d=2), \quad \Pi(x)=\frac{2 k^{2}}{\pi^{2}} \frac{j_{1}(2 k|x|)}{|x|} \quad(\text { if } d=3),
$$

where $J_{1}$ is the Bessel function of the first kind and order 1 and $j_{1}$ its spherical counterpart. Then, the following corollary immediately follows from equation (38) and the definitions of functions $\Theta$ and $\Pi$ : 
Corollary 2. With the functions $\Theta$ and $\Pi$ defined by (39a) and (40), one has

$$
\left[\Theta \star \mathcal{T}_{\mathbb{S}}^{b}\right](z)=-|\mathcal{D}| k^{4} q_{\star}[q \star \Pi](z) .
$$

The functions $\mathcal{T}_{\mathbb{S}}^{b}$ and $\Theta \star \mathcal{T}_{\mathbb{S}}^{b}$ both involve the convolution of the unknown contrast function $q$ with a function of compact support $|\xi| \in[0 ; k / \pi]$. However, since in the Fourier domain one has

$$
\mathcal{F}\left[\Theta \star \mathcal{T}_{\mathbb{S}}^{b}\right](\xi)=-|\mathcal{D}| k^{4} q_{\star} \hat{q}(\xi) \quad\left(\text { if }|\xi|<\frac{k}{\pi}\right), \quad \mathcal{F}\left[\Theta \star \mathcal{T}_{\mathbb{S}}^{b}\right](\xi)=0 \quad\left(\text { if }|\xi|>\frac{k}{\pi}\right)
$$

the convolution of $\mathcal{T}_{\mathbb{S}}^{b}$ with $\Theta$ allows to recover, up to the user-chosen factor $|\mathcal{D}| k^{4} q_{\star}$, the Fourier transform of $q$ for the spatial frequencies less than $k / \pi$. In fact, it is pointed out in [10] (p. 93, after eq. 4.21) that $\hat{q}$ is analytic (by virtue of $\hat{q}$ having compact support) and hence can in principle be recovered in all of $\mathbb{R}^{d}$ from its truncated version.

\subsection{Full scattering model}

The topological derivative exploiting far-field measurements $v^{\infty}$ of the scattered field is now formulated within the full-scattering model. In this framework, the following factorization holds for the far-field operator:

Lemma 3 ([10], Theorem 4.5). Let the far-field operator $F: L^{2}(\mathbb{S}) \rightarrow L^{2}(\mathbb{S})$ be defined by (12). Then

$$
F=H^{\star} T H
$$

with operators $H, H^{\star}$ defined by (13a) and (13b), respectively. The operator $T$ : $L^{2}(D) \rightarrow L^{2}(D)$ is defined by $T \varphi=\left(T_{b}^{-1}-S\right)^{-1} \varphi$ in terms of the operators $S: L^{2}(D) \rightarrow$ $L^{2}(D)$ and $T_{b}: L^{2}(D) \rightarrow L^{2}(D)$ appearing in $(6)$.

Proof. The proof follows $[1,10]$ and is presented for completeness. By superposition, for given $g \in L^{2}(\mathbb{S}), F g$ is the scattering far-field pattern arising from illuminating the inhomogeneity $D$ by the incident wave $H g \in L^{2}(D)$. By virtue of the LippmannSchwinger equation (6), the total field $u \in L^{2}(D)$ in $D$ solves $\left(I-S T_{b}\right) u=H g(x \in D)$. By (8), the far-field pattern corresponding to $u$ is then given by $H^{\star} T_{b} u$, i.e. by $H^{\star} T_{b}\left(I-S T_{b}\right)^{-1} H g=H^{\star}\left(T_{b}^{-1}-S\right)^{-1} H g$. Hence $F=H^{\star}\left(T_{b}^{-1}-S\right)^{-1} H$.

The topological derivatives and their decay properties are thus as follows:

Proposition 5. Under the full-scattering model, the topological derivatives $\mathcal{T}[g]$ and $\mathcal{T}_{\mathbb{S}}$ are given by

$$
\begin{aligned}
\mathcal{T}[g](z) & =-|\mathcal{D}| k^{2} q_{\star} \operatorname{Re}\left[\left(\Phi_{z}^{\infty}, g\right)_{L^{2}(\mathbb{S})}\left(T H g, H \Phi_{z}^{\infty}\right)_{L^{2}(D)}\right] \\
\mathcal{T}_{\mathbb{S}}(z) & =-|\mathcal{D}| k^{2} q_{\star} \operatorname{Re}\left[\left(H \Phi_{z}^{\infty}, T H \Phi_{z}^{\infty}\right)_{L^{2}(D)}\right]
\end{aligned}
$$

Moreover, they decay with the distance dist $(z, D)$ according to

$$
\begin{aligned}
|\mathcal{T}[g](z)| & =\mathcal{O}\left(|z|^{(1-d) / 2} \operatorname{dist}(z, D)^{(1-d) / 2}\right) & |z| \rightarrow \infty . \\
\left|\mathcal{T}_{\mathbb{S}}(z)\right| & =\mathcal{O}\left(\operatorname{dist}(z, D)^{1-d}\right) &
\end{aligned}
$$


Proof. Formulae (42a,b) result directly from applying Lemma 3 to (26a-b). Moreover, the decay properties (43) are identical to those obtained under the Born approximation and hold for the same reasons (they are not influenced by whether $T$, rather than $T_{b}$, is used in the factorization $\left.F=H^{\star} T H\right)$.

The far-field operator $F$ is normal and compact [6]. As a consequence, there exists a complete set of orthonormal eigenfunctions $\Psi_{\ell} \in L^{2}(\mathbb{S})$ with corresponding eigenvalues $\lambda_{\ell} \in \mathbb{C}$ such that

$$
F g=\sum_{\ell=0}^{\infty} \lambda_{\ell}\left(g, \Psi_{\ell}\right)_{L^{2}(\mathbb{S})} \Psi_{\ell}
$$

This allows the following reformulations of $\mathcal{T}[g]$ and $\mathcal{T}_{\mathbb{S}}$ :

Proposition 6. The topological derivative $\mathcal{T}_{\mathbb{S}}$ is given in terms of the $L^{2}(\mathbb{S})$-orthonormal system $\left(\lambda_{\ell}, \Psi_{\ell}\right)_{\ell \in \mathbb{N}}$ of $F$ by

$$
\mathcal{T}_{\mathbb{S}}(z)=-|\mathcal{D}| k^{2} \sum_{\ell=0}^{\infty} q_{\star} \operatorname{Re}\left[\lambda_{\ell}\right]\left|\left(\Phi_{z}^{\infty}, \Psi_{\ell}\right)_{L^{2}(\mathbb{S})}\right|^{2} .
$$

Moreover, letting $g=\Psi_{k}$ for some $k \in \mathbb{N}$, the topological derivative $\mathcal{T}\left[\Psi_{k}\right]$ is given by

$$
\mathcal{T}\left[\Psi_{k}\right](z)=-|\mathcal{D}| k^{2} q_{\star} \operatorname{Re}\left[\lambda_{k}^{-1}\right]\left|\left(T H \Psi_{k}, H \Phi_{z}^{\infty}\right)_{L^{2}(D)}\right|^{2} .
$$

This in particular implies, by virtue of Lemma 1 and (29), that

$$
\mathcal{T}\left[\Psi_{k}\right](z)=\mathcal{O}\left(\operatorname{dist}(z, D)^{1-d}\right) .
$$

Proof. Reformulation (45) is obtained by using (44) into (42b), while (46) is established in the same way as (28b) with $T_{b}$ replaced with $T$, taking advantage of the factorization (41).

The topological derivative $\mathcal{T}[g]$ can in fact easily be reformulated in terms of $\left(\Psi_{\ell}, \lambda_{\ell}\right)_{\ell \in \mathbb{N}}$ for arbitrary densities $g \in L^{2}(\mathbb{S})$; the resulting expression is not shown as it does not permit additional general insight. Besides, the case of a single incident plane wave is summarized in the following remark:

Remark 5. The topological derivative for the case where $D$ is probed using a single plane wave of incidence direction $\theta$, denoted $\mathcal{T}[\theta](z)$, is obtained by replacing $F g(\hat{x})$ with $A(\hat{x}, \theta)$ in $(17),(24),(26 a)$ and $\left(\Phi_{z}^{\infty}, g\right)_{L^{2}(\mathbb{S})}$ with $\Phi_{z}^{\infty}(\theta)$ in (26a). As a result, one obtains

$$
\begin{aligned}
\mathcal{T}[\theta](z) & =-|\mathcal{D}| k^{2} q_{\star} \operatorname{Re}\left[\Phi_{z}^{\infty}(\theta) H^{\star} T H \Phi_{z}^{\infty}(-\theta)\right] \\
& =\mathcal{O}\left(\operatorname{dist}(z, D)^{(1-d) / 2}\right) \quad|z| \rightarrow \infty
\end{aligned}
$$

This case is easily generalized to measurements available for a finite number $N$ of plane waves with incidence directions $\theta_{n}(1 \leq n \leq N)$, with the topological derivative at $z$ then given by $\sum_{n=1}^{N} \mathcal{T}\left[\theta_{n}\right](z)$. Unsurprisingly (since the assumed available data is scarcer), the decay of $\mathcal{T}[\theta](z)$ away from $D$ is less pronounced than that of $\mathcal{T}_{\mathbb{S}}(z)$ or $\mathcal{T}\left[\Psi_{k}\right](z)$. Moreover, $\operatorname{sign}(\mathcal{T}[\theta](z))$ cannot be ascertained from the above expression.

Propositions 5 and 6 address the decay of the topological derivative away from $D$, but not their sign properties; these are addressed next. 
Sign properties of the topological derivative. Determining the sign of the topological derivative is more difficult than in the case of the Born approximation. This has much to do with the fact that, $F$ being normal but (unlike $T_{b}$ ) not self-adjoint, the eigenvalues $\lambda_{\ell}$ are complex-valued. They are in fact known $[6,38]$ to lie on the circle of the complex $\lambda$-plane defined by

$$
k \gamma_{d}^{2}\left|\lambda_{\ell}\right|^{2}-\operatorname{Im}\left[\lambda_{\ell}\right]=0
$$

Equation (45) highlights the fact that the indicator function $z \mapsto \mathcal{T}_{\mathbb{S}}(z)$ defined in the topological derivative approach, based on an asymptotic expansion of the $L^{2}$ norm-based misfit function (16), reduces to the sum of the projections of the test function $\Phi_{z}^{\infty}$ onto the eigenvectors $\Psi_{\ell}$ of the far-field operator, weighted by the products $q_{\star} \operatorname{Re}\left[\lambda_{\ell}\right]$ with the trial contrast $q_{\star}$ chosen a priori. Equation (45) shows that the sign of $\mathcal{T}_{\mathbb{S}}$ depends on the signs of $\operatorname{Re}\left(\lambda_{\ell}\right)$, with $\mathcal{T}_{\mathbb{S}}(z)$ guaranteed to be negative if $\operatorname{sign}\left[\operatorname{Re}\left(\lambda_{\ell}\right)\right]$ is constant and equal to $\operatorname{sign}\left(q_{\star}\right)$ for all $\ell \in \mathbb{N}$. Equation (48) implies that $-8 \pi^{2} / k \leq \operatorname{Re}\left(\lambda_{\ell}\right) \leq 8 \pi^{2} / k$ and $\operatorname{Im}\left(\lambda_{\ell}\right) \geq 0$ for any $\ell \in \mathbb{N}$, allowing to readily characterize $\operatorname{sign}\left[\operatorname{Im}\left(\lambda_{\ell}\right)\right]$ but not $\operatorname{sign}\left[\operatorname{Re}\left(\lambda_{\ell}\right)\right]$ (whereas the latter sign was known in the Born approximation case). Indeed, the analytical exemple of Sec. 3.5 will show that $\operatorname{Re}\left(\lambda_{\ell}\right)$ can be either positive or negative for sufficiently high frequency and/or contrast, causing sign changes of $\mathcal{T}_{\mathbb{S}}(z)$ for $z \in D$. Likewise, the verification of the sign heuristic for $\mathcal{T}\left[\Psi_{k}\right]$ as given by (46) also requires $\operatorname{sign}\left[\operatorname{Re}\left(\lambda_{k}\right)\right]=\operatorname{sign}\left(q_{\star}\right)$. However, this requirement can be satisfied in practice by selecting the pair $\left(\lambda_{k}, \Psi_{k}\right)$ appropriately since only one such pair is involved in (46).

It is nevertheless possible to extend the validity of the sign-characterization result (33) beyond the Born approximation, to a limited extent. To this end, assume that $q$ has a constant sign over $D$ and introduce the operator $\widetilde{S}: L^{2}(D) \rightarrow L^{2}(D)$ such that $\widetilde{S}=\operatorname{sign}(q) T_{b}^{1 / 2} S T_{b}^{1 / 2}$, with the operator $T_{b}^{1 / 2}: L^{2}(D) \rightarrow L^{2}(D)$ defined by $T_{b}^{1 / 2} f=$ $\left(k^{2}|q|\right)^{1 / 2} f$. Setting $\psi:=T_{b}^{1 / 2} H \Phi_{z}^{\infty} \in L^{2}(D)$, and recalling that $T=\left(T_{b}^{-1}-S\right)^{-1}$, the topological derivative $\mathcal{T}_{\mathbb{S}}(z)$ can be recast from (42b) in the form

$$
\begin{aligned}
\mathcal{T}_{\mathbb{S}}(z)=-|\mathcal{D}| k^{2} q_{\star} \operatorname{sign}(q) \operatorname{Re}\left[\left(\psi, T_{b}^{-1 / 2}\right.\right. & \left.\left.T T_{b}^{-1 / 2} \psi\right)_{L^{2}(D)}\right] \\
& =-|\mathcal{D}| k^{2} q_{\star} \operatorname{sign}(q) \operatorname{Re}\left[\left(\psi,(I-\widetilde{S})^{-1} \psi\right)_{L^{2}(D)}\right] .
\end{aligned}
$$

The following result then holds:

Proposition 7. Assume that (i) $q$ has a constant sign over $D$, and (ii) $D, k$ and $q$ are such that $\|\widetilde{S}\|<1 / 2$, where $\|\widetilde{S}\|:=\sup _{\|\phi\|_{L^{2}(D)}=1}\|\widetilde{S} \phi\|_{L^{2}(D)}$. Then:

$$
\operatorname{sign}\left(\mathcal{T}_{\mathbb{S}}(z)\right)=-\operatorname{sign}\left(q_{\star} q\right) .
$$

Proof. Using the identity $(I-\widetilde{S})^{-1}=I+\widetilde{S}(I-\widetilde{S})^{-1}, \mathcal{T}_{\mathbb{S}}(z)$ is recast as

$$
\mathcal{T}_{\mathbb{S}}(z)=-|\mathcal{D}| k^{2} q_{\star} \operatorname{sign}(q)\left(\|\psi\|_{L^{2}(D)}^{2}+\operatorname{Re}\left[\left(\psi, \widetilde{S}(I-\widetilde{S})^{-1} \psi\right)_{L^{2}(D)}\right]\right) .
$$

Moreover, one has $\left\|(I-\widetilde{S})^{-1}\right\| \leq 1 /(1-\|\widetilde{S}\|)$ whenever $\|\widetilde{S}\|<1$. Applying the CauchySchwarz inequality to $\left|\left(\psi, \widetilde{S}(I-\widetilde{S})^{-1} \psi\right)_{L^{2}(D)}\right|$, one thus obtains

$$
\left|\left(\psi, \widetilde{S}(I-\widetilde{S})^{-1} \psi\right)_{L^{2}(D)}\right| \leq \frac{\|\widetilde{S}\|}{1-\|\widetilde{S}\|}\|\psi\|_{L^{2}(D)}^{2}
$$


The condition $\|\widetilde{S}\|<1 / 2$ therefore ensures that $\left|\left(\psi, \widetilde{S}(I-\widetilde{S})^{-1} \psi\right)_{L^{2}(D)}\right|<\|\psi\|_{L^{2}(D)}^{2}$ for any $\psi \in L^{2}(D)$. This in turn guarantees that $\|\psi\|_{L^{2}(D)}^{2}+\operatorname{Re}\left[\left(\psi, \widetilde{S}(I-\widetilde{S})^{-1} \psi\right)_{L^{2}(D)}\right]>0$, which completes the proof.

Remark 6. The condition $\|\widetilde{S}\|<1 / 2$ limits this sign-characterization result to scatterers of moderate strength, which are in particular within the applicability bounds of iterated Born (i.e. Neumann series) solution methods [39], while extending the corresponding result for the Born approximation case (for which $\|\widetilde{S}\| \ll 1$ ).

\subsection{Analytical example: spherical scatterer in $\mathbb{R}^{3}$}

Topological derivative. To illustrate the foregoing developments, consider scattering by a homogeneous spherical obstacle $D$ of unit radius and centered at the origin, so that $\partial D=\left\{x \in \mathbb{R}^{3}:|x|=1\right\}$. Assuming illumination by an incident plane wave $u_{i}=h(\cdot, \theta)$ propagating along the direction $\theta \in \mathbb{S}$, which can be expanded over the set of $L^{2}(\mathbb{S})$-orthonormal spherical harmonics $\left(Y_{\ell}^{m}\right)_{\ell \in \mathbb{N}, m \in\{-\ell, \ldots, \ell\}}$ as

$$
h(x, \theta)=\sum_{\ell=0}^{\infty} \sum_{m=-\ell}^{\ell} 4 \pi \mathrm{i}^{\ell} j_{\ell}(k|x|) Y_{\ell}^{m}(\hat{x}) \overline{Y_{\ell}^{m}}(\theta)
$$

by virtue of the Jacobi-Anger identity and the Legendre addition theorem (see e.g. eqs. 10.60.7 and 14.30.9 in [33]). The total field $u$ in $D$ and the scattered field $v$ in $\mathbb{R}^{3} \backslash D$ that together solve the forward scattering problem $(1 \mathrm{a}-\mathrm{c})$ can be similarly expanded as

$$
\begin{aligned}
& u(x, \theta)=\sum_{\ell=0}^{\infty} \sum_{m=-\ell}^{\ell} u_{\ell}^{m}(\theta) j_{\ell}(n k|x|) Y_{\ell}^{m}(\hat{x}) \quad \text { for } x \in D, \theta \in \mathbb{S}, \\
& v(x, \theta)=\sum_{\ell=0}^{\infty} \sum_{m=-\ell}^{\ell} v_{\ell}^{m}(\theta) h_{\ell}(k|x|) Y_{\ell}^{m}(\hat{x}) \quad \text { for } x \in \mathbb{R}^{3} \backslash D, \theta \in \mathbb{S},
\end{aligned}
$$

where $n=\sqrt{1+q}, j_{\ell}$ and $h_{\ell}$ denote respectively the $p$ th-order spherical Bessel and Hankel functions of the first kind. On using the transmission conditions $u=u_{i}+v$ and $n \partial_{|x|} u=\partial_{|x|}\left(u_{i}+v\right)$ on $\partial D$ and the $L^{2}(\mathbb{S})$-orthonormality of spherical harmonics, the solution for $v$ in $\mathbb{R}^{d} \backslash D$ is found to be given by

$$
v(x, \theta)=4 \pi \sum_{\ell=0}^{\infty} \sum_{m=-\ell}^{\ell} \mathrm{i}^{\ell} \Lambda_{\ell}(q, k) h_{\ell}(k|x|) Y_{\ell}^{m}(\hat{x}) \overline{Y_{\ell}^{m}}(\theta),
$$

with the coefficients $\Lambda_{\ell}(q, k)$ given by

$$
\Lambda_{\ell}(q, k)=\frac{j_{\ell}(n k) j_{\ell}^{\prime}(k)-n j_{\ell}^{\prime}(n k) j_{\ell}(k)}{n j_{\ell}^{\prime}(n k) h_{\ell}(k)-j_{\ell}(n k) h_{\ell}^{\prime}(k)}
$$

( $f^{\prime}$ denoting the derivative of $f$ with respect to its argument). Note that $\Lambda_{\ell}(q, k)$ is nonsingular, as the denominator $n j_{\ell}^{\prime}(n k) h_{\ell}(k)-j_{\ell}(n k) h_{\ell}^{\prime}(k)$ can be shown to be nonzero for any $k \in \mathbb{R}^{+}$and $\ell \in \mathbb{N}$ (see e.g. [40]). Using equation (50) and Theorem 2.15 of [5], the scattered far-field pattern generated by a plane wave impinging on the unit penetrable 
ball centered at the origin is thus given by

$$
v^{\infty}(\hat{x}, \theta)=\frac{16 \pi^{2}}{\mathrm{i} k} \sum_{\ell=0}^{\infty} \Lambda_{\ell}(q, k) Y_{\ell}^{m}(\hat{x}) \overline{Y_{\ell}^{m}}(\theta) .
$$

Then, since the spherical harmonics $Y_{\ell}^{m}$ constitute an orthonormal system for $L^{2}(\mathbb{S})$, one concludes from definition (12) that the eigenvalues of the far-field operator $F$ are given by

$$
\lambda_{\ell}^{m}=\frac{16 \pi^{2}}{\mathrm{i} k} \Lambda_{\ell}(q, k) \quad \text { for } \ell \in \mathbb{N}, m \in\{-\ell, \ldots, \ell\}
$$

with the associated eigenfunctions $\Psi_{\ell}^{m} \equiv Y_{\ell}^{m}$, counting multiplicity. Note that eq. (48) implies that $\Lambda_{\ell}$ satisfy $\left|\Lambda_{\ell}\right|^{2}+\operatorname{Re}\left[\Lambda_{\ell}\right]=0$ for any $\ell \in \mathbb{N}$. The latter identity is also easily checked directly from definition (50) of $\Lambda_{\ell}$ and the fact that $j_{\ell}=\operatorname{Re}\left[h_{\ell}\right]$. Finally, on applying the Jacobi-Anger expansion (49) to $\Phi_{z}^{\infty}(\hat{x})=\overline{h(z, \hat{x})}$, using again the orthonormality of the $Y_{\ell}^{m}$ and invoking the identity $\sum_{m=-\ell}^{m=\ell} Y_{\ell}^{m}(\hat{z}) \overline{Y_{\ell}^{m}(\hat{z})}=(2 \ell+1) / 4 \pi$ (a special case of the Legendre addition theorem), the topological derivative $\mathcal{T}_{\mathbb{S}}$ is found from (45) to be given by

$$
\mathcal{T}_{\mathbb{S}}(z)=-64 \pi^{3} k q_{\star}|\mathcal{D}| \sum_{\ell=0}^{\infty}(2 \ell+1) \operatorname{Im}\left[\Lambda_{\ell}(q, k)\right] j_{\ell}(k|z|)^{2} .
$$

One can show from well-known limiting forms of the spherical Bessel functions (see e.g. [33], Chap. 10) that the coefficients $\Lambda_{\ell}(q, k)$ admit the low-frequency expansion

$$
\Lambda_{\ell}(q, k)=\mathrm{i} q \frac{k^{2 \ell+3}}{(2 \ell+1) ! !(2 \ell+3) ! !}\left(1+\mathcal{O}\left(k^{2}\right)\right)
$$

(where $n ! !=1 \times 3 \times \ldots n$ for any odd integer $n$ ) and the large-order expansion

$$
\Lambda_{\ell}(q, k)=\mathrm{i} q \frac{k^{3}}{16 \ell^{3}}\left(\frac{e k}{2 \ell}\right)^{2 \ell}\left(1+\mathcal{O}\left(\ell^{-1}\right)\right) .
$$

Both limiting cases are consistent with the sign heuristic of the topological derivative.

Results. This section provides some numerical results illustrating the behavior of the topological derivative (52) with $q_{\star}=q$. For convenience of presentation, a normalization defined by

$$
\widetilde{\mathcal{T}}(z)=\left(\max _{z}(|\mathcal{T}(z)|)\right)^{-1} \mathcal{T}(z)
$$

is applied to $\mathcal{T}=\mathcal{T}_{\mathbb{S}}$, and the rescaled version $\widetilde{\mathcal{T}}_{\mathbb{S}}$ is plotted for each example as a function of the distance $|z| \in[0 ; 4]$ to the center of $D$.

The first example assumes $q=10^{-4}$ and $k=10$, i.e. is well within the Born approximation. Figure 1a shows the sharp decrease of $\left|\Lambda_{\ell}\right|$ as $\ell$ increases, which justifies the approximate evaluation of the infinite series (52) at an appropriate truncation level $\ell_{0}$ (the examples of this section required $\ell_{0}=120$ at most). The largest negative values of $\mathcal{T}_{\mathbb{S}}$ occur inside $D$, as expected from the analysis of Section 3.3 (Fig. 1b).

In the next two examples (Figures 2 and 3), the parameters $q$ and $k$ are chosen so that the configurations correspond to limit cases in terms of the validity of the Born 


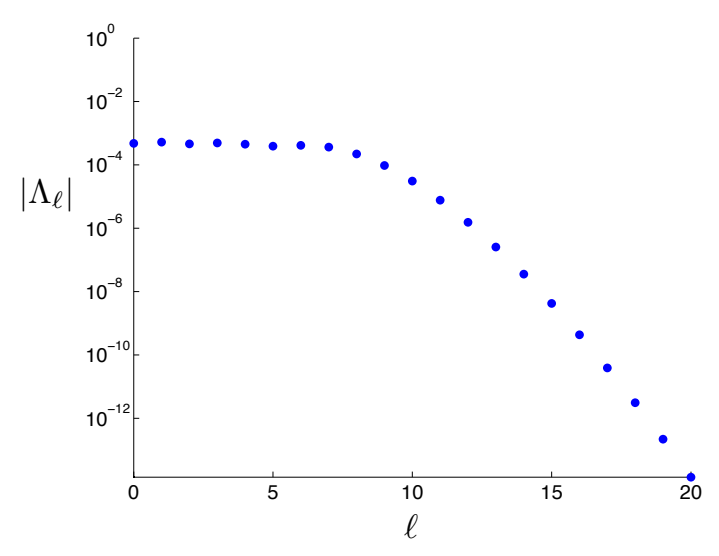

(a) Eigenvalues of $F$

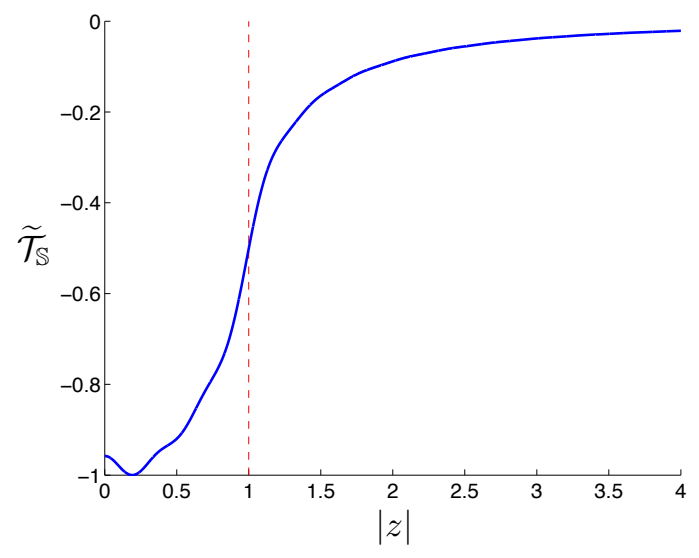

(b) Radial plot of $\mathcal{T}_{\mathbb{S}}(z)$

Figure 1. Unit spherical obstacle with $q=10^{-4}$ and $k=10$.

approximation. The eigenvalue sequences $\left\{\Lambda_{\ell}\right\}$, plotted in the complex plane on Figs. 2a and 3 a (using colored dots, the color scale indicating the value of their order $\ell$ ), are seen to accumulate at the origin in accordance with their large-order behavior, and also to lie on a circle as predicted by (48). However, the behavior of $\mathcal{T}_{\mathbb{S}}$ in these two cases is clearly different. In the first case, where $q=1.510^{-2}>0$ (Fig. 2), one has $\operatorname{Im}\left[\Lambda_{\ell}\right]>0$ for all $\ell \in \mathbb{N}$, which ensures that $\widetilde{\mathcal{T}}_{\mathbb{S}}(z)<0$ for all $z \in \mathbb{R}^{3}$ since $\operatorname{sign}\left(q_{\star} q\right)=1$; moreover, Fig. 2b shows that $\operatorname{sign}\left(q_{\star} q\right)=1$ attains pronounced negative values for $|z|<1$, i.e. inside $D$. In the second case, where $q=810^{-2}$, Fig. 3a shows that the sequence $\left\{\operatorname{Im}\left[\Lambda_{\ell}\right]\right\}$, and thus $\left\{\operatorname{Re}\left[\lambda_{\ell}\right]\right\}$, has sign changes. Moreover, $\widetilde{\mathcal{T}}_{\mathbb{S}}(z)$, while being predominantly negative inside $D$ (and hence acceptably consistent with the original sign heuristic), also has sign changes. In both cases, $\left|\widetilde{\mathcal{T}}_{\mathbb{S}}(z)\right|$ decays as predicted away from $D$.

Validity of the sign heuristic. The decay of $\left|\mathcal{T}_{\mathbb{S}}(z)\right|$ away from $D$ is characterized by (30) and (43), respectively, for the Born approximation and the full scattering

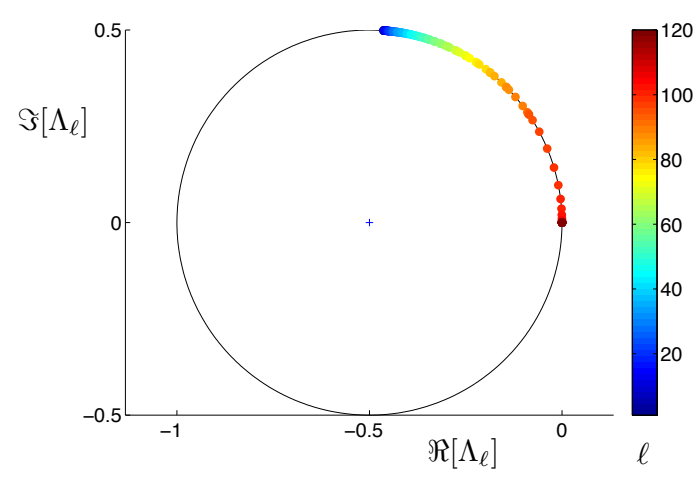

(a) Eigenvalues of $F$ in the complex plane

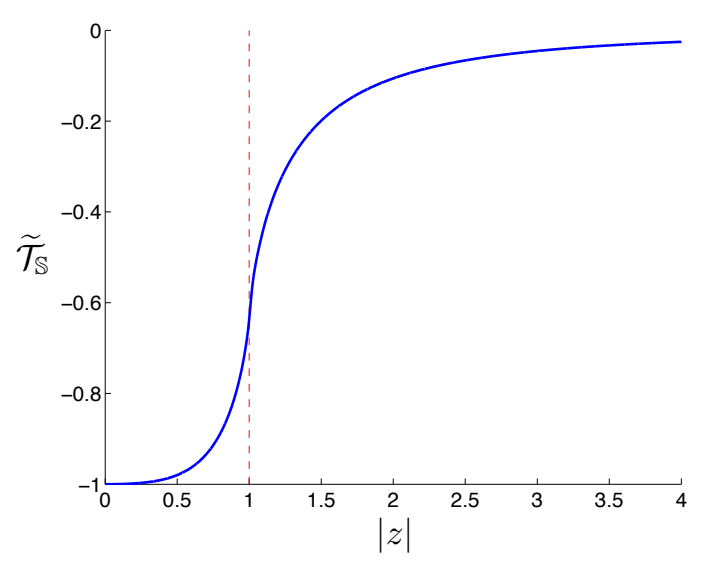

(b) Radial plot of $\mathcal{T}_{\mathbb{S}}(z)$

Figure 2. Identification of a unit spherical obstacle $\left(q=1.510^{-2}, k=100\right)$. 


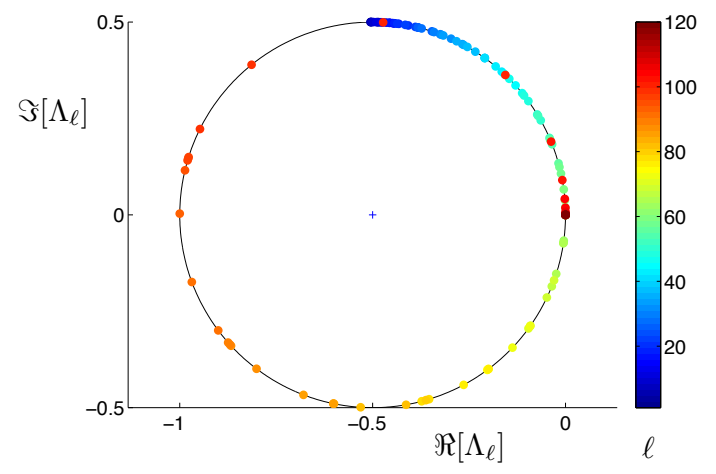

(a) Eigenvalues of $F$ in the complex plane

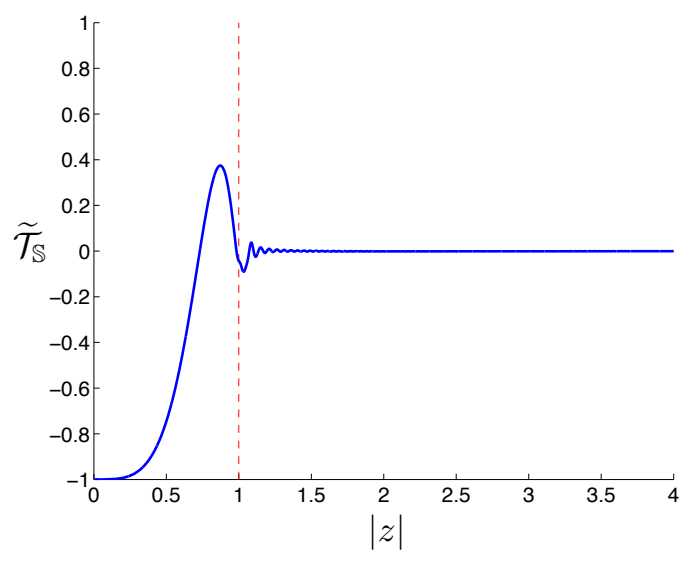

(b) Radial plot of $\mathcal{T}_{\mathbb{S}}(z)$

Figure 3. Identification of a unit spherical obstacle $\left(q=810^{-2}, k=100\right)$.

model, with the interpretation of the sign of $\mathcal{T}_{\mathbb{S}}(z)$ remaining an open question in the latter case when Proposition 7 does not apply. Nonetheless, as emphasized by (45), the sign heuristic of the method is satisfied whenever $\operatorname{Re}\left(\lambda_{\ell}\right)$ all have the same sign, and may also be satisfied in other cases. If available measurements are sufficient for constructing the operator $F$, its eigenvalues are computable from the available data and their signs checkable. Moreover, as illustrated by the previously shown numerical results, satisfactory reconstructions are still achievable in cases where $\operatorname{sign}\left[\operatorname{Re}\left(\lambda_{\ell}\right)\right]$ is not constant (as in Fig. 3).

To investigate further the sign heuristic on the present analytical example, the average sign $\langle S\rangle$ defined as a function of $q$ and $k$ by

$$
\langle S\rangle(q, k)=\frac{1}{\ell_{\max }} \sum_{\ell=0}^{\ell_{\max }} \operatorname{sign}\left(\operatorname{Im}\left[\Lambda_{\ell}(q, k)\right]\right)
$$

with the truncation parameter $\ell_{\max }(q, k)<200$ set such that $\operatorname{Im}\left[\Lambda_{\ell}(q, k)\right]<10^{-20}$ for all $\ell>\ell_{\max }$, is computed. One has $-1 \leq\langle S\rangle(q, k) \leq 1$ by construction, with $\langle S\rangle(q, k)=1$ indicating perfect verification of the sign heuristic. The function $\langle S\rangle$ is plotted in Figure 4, with the validity limits of the Born approximation in the high- and low-frequency regimes (as defined by (73) and (74)) indicated by dashed lines and the configurations corresponding to Figures 1-3 indicated by symbols. This plot indicates that $\langle S\rangle(q, k)=1$ in a parameter region outside that defined by Proposition 7 (and hence also beyond the Born approximation), in which the validity of the sign heuristic is thus corroborated empirically.

\subsection{Numerical examples in $\mathbb{R}^{2}$}

In this section, numerical results corresponding to the identification of a set of homogeneous scattering obstacles (i.e $q$ is piecewise-constant and $D=\operatorname{supp}(q-1)$ ) embedded in $\mathbb{R}^{2}$ are presented. The forward full scattering model is implemented 


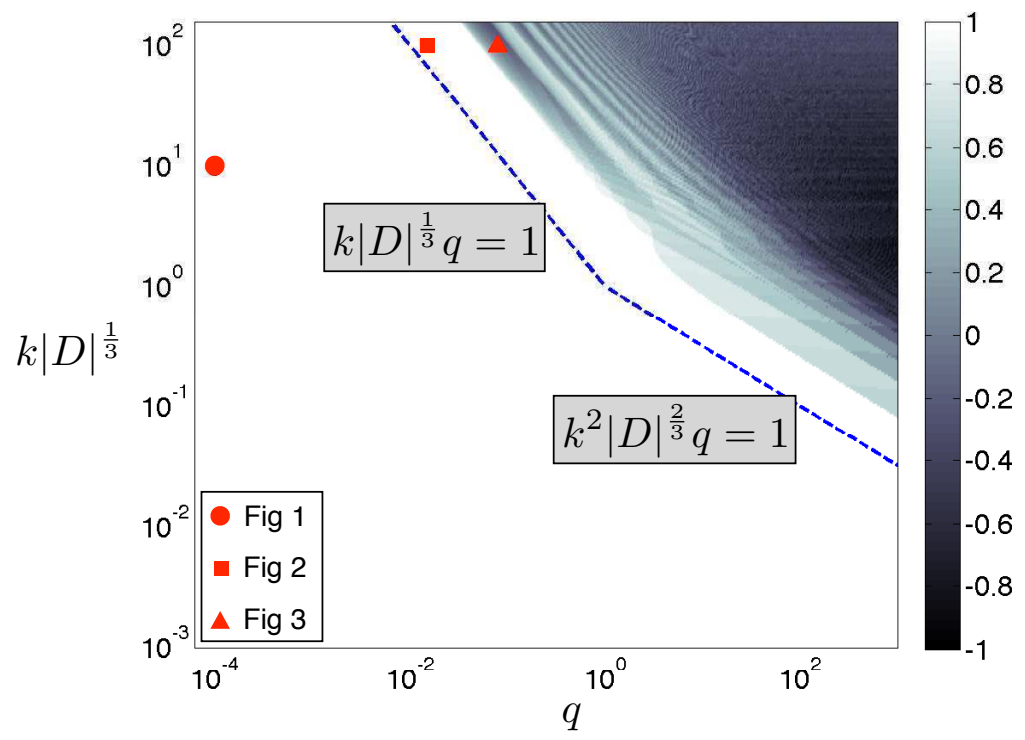

Figure 4. Average sign $\mathcal{S}(q, k)$ of the eigenvalues of the far-field operator.

via a numerical solution of the Lippmann-Schwinger integral equation (6). The discretization method proposed in [41] is used, with the discretization length $h$ adjusted to the wavelength according to $h=\lambda / 10=\pi / 5 k$. Given a set of $N=60$ plane waves with $k=2$ and equally-spaced incident directions $\theta_{j}$ on $\mathbb{S}$ (with $\theta_{j}=$ $(\cos (2 \pi(j-1) / N), \sin (2 \pi(j-1) / N))$ for $j=1, \ldots, N)$, synthetic measurements of the scattered far-field pattern (9) are generated for each configuration considered in order to compute the corresponding far-field operator (12). The topological derivative (42b) is then computed and its rescaled counterpart (53) is finally plotted (see Figures 5, 6 and 7 ) over the sampling region $z \in[-10 ; 10] \times[-10 ; 10]$.

Figure 5 shows that satisfactory results are obtained for the identification of either a single L-shaped scatterer (left) or a set of two obstacles (one circular, one L-shaped),
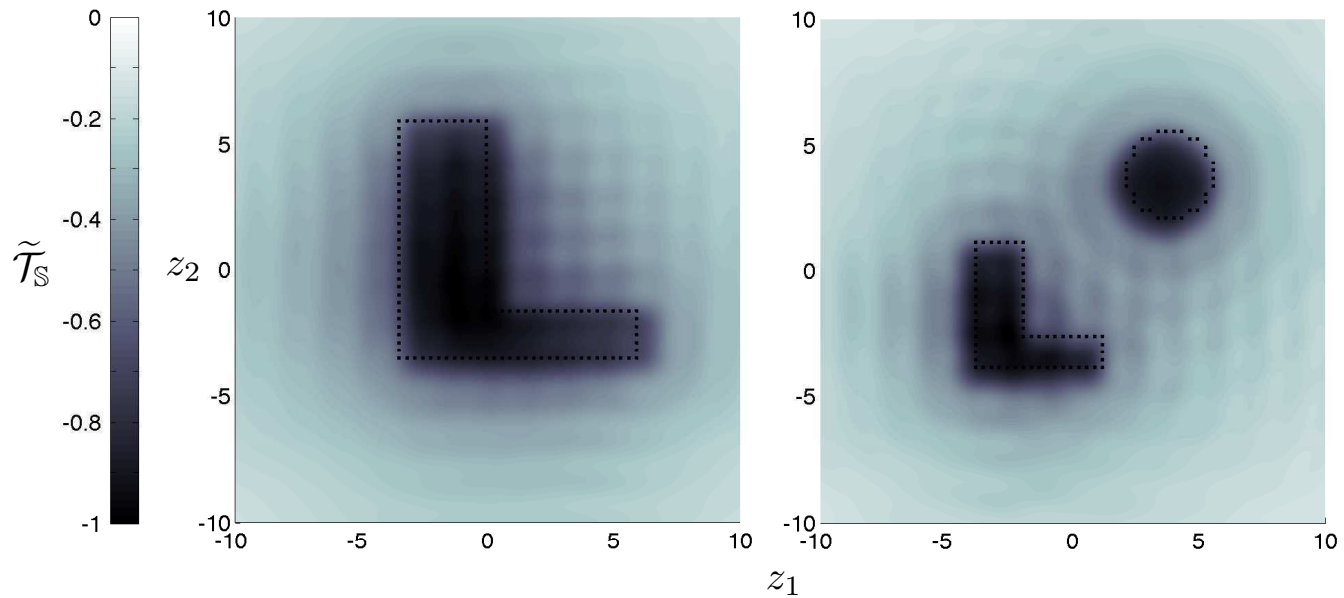

Figure 5. Identification of an inhomogeneous medium (dashed contour) with one (left) or two (right) components characterized by $q=0.1$ and using $q_{\star}=0.1$. 


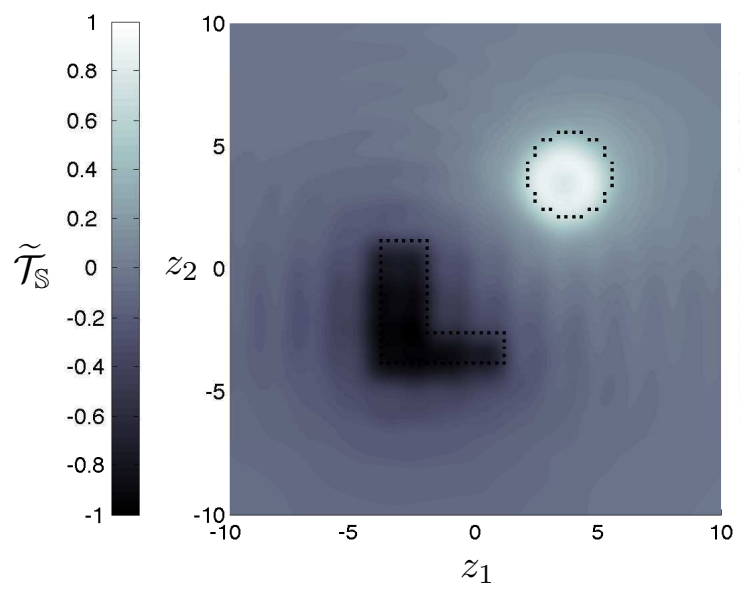

Figure 6. Identification of two scatterers respectively characterized by $q_{1}=0.1$ (lower left) and $q_{2}=-0.1$ (upper right), using $q_{\star}=0.1$.
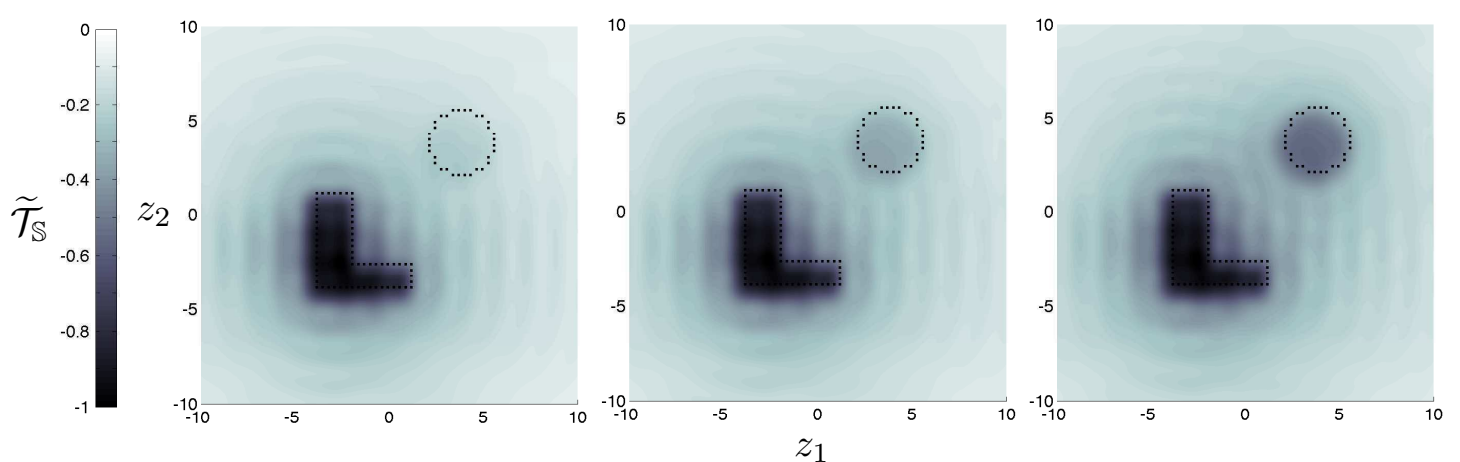

Figure 7. Identification of two scatterers characterized by $q_{1}=0.1$ (lower left) and $q_{2}=\{0.01 ; 0.025 ; 0.05\}$ (upper right) and using $q_{\star}=0.1$.

with the negative values of $\widetilde{\mathcal{T}}_{\mathbb{S}}(z)$ closest to -1 occurring in both cases in or near $D$. In particular, the two unknown obstacles are well resolved in Figure 5 (right). On Figure 6, the scatterer $D$ considered has two homogeneous components characterized by $q_{1}=0.1$ and $q_{2}=-0.1$, and $\mathcal{T}_{\mathbb{S}}$ is computed with $q_{\star}=q_{1}$. The locations and supports of the obstacles are well identified. Moreover, $\widetilde{\mathcal{T}}_{\mathbb{S}}(z)$ changes its sign from one object to the other as expected from the analysis of Secs. 3.3 and 3.4, with its most pronounced negative values occurring in the support of the scatterer for which $\operatorname{sign}\left(q_{\star} q\right)=1$. Finally, the identification of two objects with contrasts $q_{1}, q_{2}$ of the same sign is shown in Figure 7 for three values $q_{2} / q_{1}=0.01,0.025,0.05$ of the contrast ratio (with $q_{1}=0.1$ in all cases). The results suggest that the best reconstructions are achieved when $q_{1}$ and $q_{2}$ have similar values. 


\section{Inverse scattering by point-like obstacles}

\subsection{Direct scattering problem and topological derivative}

The analysis developed in Section 3 can be carried over to small, point-like scatterers embedded in a homogeneous background medium. Such configurations define a simple, yet insightful, framework for further comparison with some of the sampling methods mentioned in Section 1. In this context, the topological derivative is closely related to a broader class of asymptotic methods [14] where geometrical information on small targets is recovered using asymptotic expansions of the forward solution. Such asymptotic analyses have been used in a number of studies for providing mathematical justifications to several imaging methodologies, in particular time-reversal and DORT [42, 43, 44], MUSIC-type algorithms [45, 46, 47] and reverse-time migration [48].

\subsection{Born approximation}

Let $D=D^{\delta}$ denote a set of $M$ point-like scatterers characterized by a common scaling size parameter $\delta>0$, i.e. $D_{m} \equiv D_{m}^{\delta}:=y_{m}+\delta \mathcal{D}_{m}$ with centers $y_{m} \in \mathbb{R}^{d}$, normalized shapes $\mathcal{D}_{m} \subset \mathbb{R}^{d}$ and real-valued constant contrasts $q_{m}, m=1, \ldots, M$. Besides, let

$$
a:=\min _{1 \leq m<n \leq M}\left|y_{m}-y_{n}\right|
$$

denote the minimal distance between the scatterers. Assuming illumination by the incident plane wave $u_{i}=h(\cdot, \theta)$ (see $(3)$ ), the corresponding scattered field reduces to sums of asymptotic formulae (20) with $z$ replaced by $y_{m}$ and $\varepsilon$ by $\delta$, i.e.:

$$
v(x, \theta)=\sum_{m=1}^{M} Q_{m} k^{2} u_{i}\left(y_{m}, \theta\right) \Phi\left(x, y_{m}\right)+o\left(\delta^{d}\right)
$$

where $Q_{m}:=\delta^{d}\left|\mathcal{D}_{m}\right| q_{m}$ is the reflectivity of the $m$-th obstacle, while the kernel $A(\hat{x}, \theta)$ of the far-field operator is given by

$$
A(\hat{x}, \theta)=A^{0}(\hat{x}, \theta)+o\left(\delta^{d}\right)=\sum_{m=1}^{M} A_{m}^{0}(\hat{x}, \theta)+o\left(\delta^{d}\right),
$$

where, using (23), $A_{m}^{0}(\hat{x}, \theta)$ is given by $A_{m}^{0}(\hat{x}, \theta)=k^{2} Q_{m} \overline{\Phi_{y_{m}}^{\infty}(\theta)} \Phi_{y_{m}}^{\infty}(\hat{x})$. In particular, the kernel $A^{0}(\hat{x}, \theta)$ thus defined is seen to be degenerate, of finite rank at most $M$. The leading-order small-scatterer asymptotic model (55) is a Born approximation in that it neglects multiple scattering and the far field is explicitly given in terms of the incident field at the obstacle locations $y_{m}$.

For each point-like obstacle, define the Herglotz operator $H_{m}: L^{2}(\mathbb{S}) \rightarrow \mathbb{C}$, with adjoint $H_{m}^{\star}: \mathbb{C} \rightarrow L^{2}(\mathbb{S})$, by

$$
H_{m} g:=\int_{\mathbb{S}} h\left(y_{m}, \theta\right) g(\theta) \mathrm{d} S_{\theta}, \quad H_{m}^{\star} f(\hat{x}):=h\left(y_{m},-\hat{x}\right) f
$$

and let $H: L^{2}(\mathbb{S}) \rightarrow \mathbb{C}^{M}$, with adjoint $H^{\star}: \mathbb{C}^{M} \rightarrow L^{2}(\mathbb{S})$, collect all $H_{m}$, i.e.

$$
H g:=\left\{H_{1} g, \ldots, H_{M} g\right\}^{\mathrm{T}}, \quad H^{\star} \boldsymbol{f}(\hat{x})=\sum_{m=1}^{M} H_{m}^{\star} f_{m}(\hat{x}) \quad\left(\boldsymbol{f}=\left\{f_{1}, \ldots, f_{M}\right\}^{\mathrm{T}} \in \mathbb{C}^{M}\right)
$$


Then, using (56), the far-field operator (12) has the expansion and factorization

$$
F=F^{0}+o\left(\delta^{d}\right), \quad F^{0}=H^{\star} \boldsymbol{T}_{b} H
$$

with $\boldsymbol{T}_{b}=k^{2} \operatorname{diag}\left(Q_{1}, \ldots, Q_{M}\right) \in \mathbb{C}^{M, M}$. Substituting (59) into (26b), the topological derivative $\mathcal{T}_{\mathbb{S}}$ is then found (using Lemma 1 for the second equality) to be given by

$$
\mathcal{T}_{\mathbb{S}}^{b}(z)=-k^{2} q_{\star}|\mathcal{D}| \operatorname{Re}\left[\left(H \Phi_{z}^{\infty}\right)^{\star} \boldsymbol{T}_{b} H \Phi_{z}^{\infty}\right]=-k^{4} q_{\star}|\mathcal{D}| \sum_{m=1}^{M} Q_{m} \zeta_{0}^{2}\left(z-y_{m}\right) .
$$

The magnitude $\left|\mathcal{T}_{\mathbb{S}}^{b}(z)\right|$ of $\mathcal{T}_{\mathbb{S}}^{b}$ hence (i) peaks at each location $y_{m}$ and (ii) has a $\mathcal{O}\left(\operatorname{dist}\left(z, D^{\delta}\right)^{1-d}\right)$ decay away from $D^{\delta}$. In addition, similarly to Section 3.3, one has $\operatorname{sign}\left[\mathcal{T}_{\mathbb{S}}^{b}\left(y_{m}\right)\right]=-\operatorname{sign}\left(q_{\star} Q_{m}\right)$ if either $M=1$ or all reflectivities $Q_{m}$ have the same sign; moreover, a counterpart to Corollary 1 can easily be established from (60), to show that $\operatorname{sign}\left[\mathcal{T}_{\mathbb{S}}^{b}\left(y_{m}\right)\right]=-\operatorname{sign}\left(q_{\star} Q_{m}\right)$ also holds when the scatterers are well separated (i.e. for large enough $k a$ ). As a result, $\mathcal{T}_{\mathbb{S}}^{b}(z)$ permits a satisfactory identification of the locations $y_{m}$ of a set of well-separated point-like scatterers.

In addition, the far-field operator $F^{0}$ is known (as a special case of [44], Theorem 4.7) to be such that

$$
F^{0} h\left(y_{m}, \cdot\right)=4 \pi k^{2} Q_{m} h\left(y_{m}, \cdot\right)+o\left((k a)^{-1}\right) .
$$

Moreover, the $M$ functions $h\left(y_{m}, \cdot\right)$ are linearly independent ([44], Proposition 13). Since the rank of $F^{0}$ is at most $M$, the eigensystem $\left(\lambda_{\ell}, \Psi_{\ell}\right)_{\ell \geq 1}$ of $F^{0}$ is approximately (in the sense of the above expansion) such that $\lambda_{m}:=4 \pi k^{2} Q_{m}$ are its only nonzero eigenvalues, with corresponding eigenfunctions $\Psi_{m}:=h\left(y_{m}, \cdot\right)$. The topological derivative $\mathcal{T}^{b}\left[\Psi_{m}\right]$ corresponding to the illumination of $D^{\delta}$ with the single incident field $H \Psi_{m}$ is, by virtue of (28a) and using $\left(\Psi_{m}, \Phi_{z}^{\infty}\right)_{L^{2}(D)}=\zeta_{0}\left(y_{m}-z\right)$, given by

$$
\mathcal{T}^{b}\left[\Psi_{m}\right](z)=-|\mathcal{D}| k^{2} q_{\star} \lambda_{m}\left|\left(\Psi_{m}, \Phi_{z}^{\infty}\right)_{L^{2}(D)}\right|^{2}=-4 \pi|\mathcal{D}| k^{4} q_{\star} Q_{m} \zeta_{0}^{2}\left(y_{m}-z\right) .
$$

Hence, $\mathcal{T}^{b}\left[\Psi_{m}\right](z)$ is seen to focus selectively on the obstacle $D_{m}^{\delta}$. Moreover, $\mathcal{T}_{\mathbb{S}}^{b}(z)$ given by (60) is such that

$$
4 \pi \mathcal{T}_{\mathbb{S}}^{b}(z)=\sum_{m=1}^{M} \mathcal{T}^{b}\left[\Psi_{m}\right](z)
$$

consistently with the fact that the $H \Psi_{m}$ are the only incident fields that produce nonzero far-field patterns when scattered by $D^{\delta}$.

\subsection{Multiple scattering using the Foldy-Lax model}

Again assuming here illumination by the incident plane wave $u_{i}=h(\cdot, \theta)$, the FoldyLax model $[49,50,51,52]$ accounts for multiple scattering in an approximate way, by assuming the scattered field $v(\cdot ; \theta)=u-u_{i}(\cdot ; \theta)$ to be given in terms of its Foldy-Lax approximation $v^{\mathrm{FL}}$ :

$$
v(x, \theta) \approx v^{\mathrm{FL}}(x, \theta), \quad v^{\mathrm{FL}}(x, \theta):=\sum_{m=1}^{M} Q_{m} k^{2} u^{\mathrm{FL}}\left(y_{m}, \theta\right) \Phi\left(x, y_{m}\right),
$$


where $Q_{m}$ are the obstacle reflectivities and $u^{\mathrm{FL}}\left(y_{m}, \theta\right)$ are determined for given $\theta$ by enforcing the self-consistency conditions

$$
u^{\mathrm{FL}}\left(y_{m}, \theta\right)=u_{i}\left(y_{m}, \theta\right)+\sum_{\substack{n=1 \\ n \neq m}}^{M} k^{2} Q_{n} u^{\mathrm{FL}}\left(y_{n}, \theta\right) \Phi\left(y_{m}, y_{n}\right), \quad(m=1, \ldots, M) .
$$

On introducing the matrix $\boldsymbol{S} \in \mathbb{C}^{M \times M}$ and the vector-valued functions $\boldsymbol{u}^{i}, \boldsymbol{u}^{\mathrm{FL}}: L^{2}(\mathbb{S}) \rightarrow$ $\left(L^{2}(\mathbb{S})\right)^{M}$ defined componentwise by

$$
\begin{aligned}
& S_{m n}=\left(1-\delta_{m n}\right) \Phi\left(y_{m}, y_{n}\right) \quad(m, n=1, \ldots, M), \\
& u_{m}^{i}(\theta)=h\left(y_{m}, \theta\right), \quad u_{m}^{\mathrm{FL}}(\theta)=u^{\mathrm{FL}}\left(y_{m}, \theta\right) \quad(m=1, \ldots, M),
\end{aligned}
$$

where $\delta_{n m}$ is the Kronecker symbol, the self-consistency conditions (63) for given incidence direction $\theta \in \mathbb{S}$ written in matrix form reads $\left(\boldsymbol{I}_{M}-\boldsymbol{S T}_{b}\right) \boldsymbol{u}^{\mathrm{FL}}(\theta)=\boldsymbol{u}^{i}(\theta)$, with $\boldsymbol{I}_{M}$ denoting the $M \times M$ identity matrix and $\boldsymbol{T}_{b}=k^{2} \operatorname{diag}\left(Q_{1}, \ldots, Q_{M}\right)$. With these notations, the far-field pattern associated with the Foldy-Lax model (62) is given by

$$
v^{\mathrm{FL}, \infty}(\hat{x}, \theta)=H^{\star}\left[\boldsymbol{T}_{b}\left(\boldsymbol{I}_{M}-\boldsymbol{S} \boldsymbol{T}_{b}\right)^{-1} \boldsymbol{u}^{i}(\theta)\right](\hat{x}) .
$$

The following result then holds:

Lemma 4. The far-field operator $F^{F L}$, defined by (12) with kernel $v^{F L, \infty}$ given by (65), has the factorization

$$
F^{F L}=H^{\star} \boldsymbol{T}^{F L} H
$$

where the Herglotz operator $H$ is defined by (58) and the matrix $\boldsymbol{T}^{F L} \in \mathbb{C}^{M \times M}$ is defined by $\boldsymbol{T}^{F L}=\boldsymbol{T}_{b}\left(\boldsymbol{I}_{M}-\boldsymbol{S T}_{b}\right)^{-1}=\left(\boldsymbol{T}_{b}^{-1}-\boldsymbol{S}\right)^{-1}$, with $\boldsymbol{T}_{b}=k^{2} \operatorname{diag}\left(Q_{1}, \ldots, Q_{M}\right)$ and $\boldsymbol{S}$ given by (64).

Proof. Definition (58) of $H$ implies that

$$
H g=\int_{\mathbb{S}} \boldsymbol{u}^{i}(\theta) g(\theta) \mathrm{d} S_{\theta}
$$

Evaluating $F^{\mathrm{FL}} g$ for some density $g \in L^{2}(\mathbb{S})$ using $(65)$ and the above identity, one thus finds

$$
F^{\mathrm{FL}} g(\hat{x})=\int_{\mathbb{S}} v^{\mathrm{FL}, \infty}(\hat{x}, \theta) g(\theta) \mathrm{d} S_{\theta}=H^{\star} \boldsymbol{T}^{\mathrm{FL}} H g(\hat{x})
$$

Substituting (66) into (26b), the topological derivative of (16) with data $v_{\mathrm{obs}}^{\infty} \equiv$ $v^{\mathrm{FL}, \infty}$ resulting from the Foldy-Lax model (62) is then found to be given by

$$
\mathcal{T}_{\mathbb{S}}^{\mathrm{FL}}(z)=-k^{2} q_{\star}|\mathcal{D}| \operatorname{Re}\left[\left(H \Phi_{z}^{\infty}\right)^{\star} \boldsymbol{T} H \Phi_{z}^{\infty}\right] .
$$

Assume that all obstacle reflectivities have the same sign, and let $\sigma=\operatorname{sign}\left(Q_{1}\right)=\ldots=$ $\operatorname{sign}\left(Q_{M}\right)$. Define the matrices $\boldsymbol{T}_{b}^{1 / 2}=k \operatorname{diag}\left(\sqrt{\left|Q_{1}\right|}, \ldots, \sqrt{\left|Q_{M}\right|}\right) \in \mathbb{C}^{M \times M}$, so that one has $\boldsymbol{T}_{b}=\sigma\left(\boldsymbol{T}_{b}^{1 / 2}\right)^{2}$, and $\widetilde{\boldsymbol{S}}=\sigma \boldsymbol{T}_{b}^{1 / 2} \boldsymbol{S} \boldsymbol{T}_{b}^{1 / 2}$. Setting $\boldsymbol{\Psi}_{z}:=\boldsymbol{T}_{b}^{1 / 2} H \Phi_{z}^{\infty} \in \mathbb{C}^{M}$, the topological derivative $\mathcal{T}_{\mathbb{S}}^{\mathrm{FL}}(z)$ can then be recast in the form

$$
\mathcal{T}_{\mathbb{S}}^{\mathrm{FL}}(z)=-k^{2} q_{\star} \sigma|\mathcal{D}| \operatorname{Re}\left[\boldsymbol{\Psi}_{z}^{\star}(\boldsymbol{I}-\widetilde{\boldsymbol{S}})^{-1} \boldsymbol{\Psi}_{z}\right]
$$

Then, the following counterpart of Proposition 7 holds: 
Proposition 8. If $Q_{1}, \ldots, Q_{m}$ are such that (i) $\operatorname{sign}\left(Q_{1}\right)=\ldots=\operatorname{sign}\left(Q_{M}\right)=\sigma$ and (ii) $\|\widetilde{\boldsymbol{S}}\|<1 / 2$ (where $\|\cdot\|$ is the matrix norm induced by the 2-norm in $\mathbb{C}^{M}$ ), then

$$
\operatorname{sign}\left(\mathcal{T}_{\mathbb{S}}^{F L}(z)\right)=-\sigma \operatorname{sign}\left(q_{\star}\right) .
$$

Proof. The proof is essentially identical to that of Proposition 7, with operator $\widetilde{S}$ replaced with matrix $\widetilde{\boldsymbol{S}}$ and norm definitions adjusted accordingly.

\subsection{The case of discrete far-field measurements}

The developments of Sections 4.2 and 4.3 can be repeated for the case where discrete far-field measurements at $N$ angular locations $\hat{x}=\theta_{n} \in \mathbb{S}$ are available for a discrete set of incident plane waves propagating along the same directions $\theta_{n}$, instead of continuous measurements for a continuous set of incidence directions. The main modifications consist in setting discrete counterparts of the Herglotz operator $H$ and the far-field operator $F$. The former is the matrix $\boldsymbol{H} \in \mathbb{C}^{M \times N}$ such that $H_{m n}:=h\left(y_{m}, \theta_{n}\right)$. The latter is the matrix $\boldsymbol{F}^{b} \in \mathbb{C}^{N \times N}$ (for the Born appproximation) or $\boldsymbol{F}^{\mathrm{FL}} \in \mathbb{C}^{N \times N}$ (for the Foldy-Lax model), respectively defined by $F_{\ell n}^{b}=v^{b, \infty}\left(\theta_{\ell}, \theta_{n}\right)$ with $v^{b, \infty}$ given by (56) and $F_{\ell n}^{\mathrm{FL}}=v^{\mathrm{FL}, \infty}\left(\theta_{\ell}, \theta_{n}\right)$ with $v^{\mathrm{FL}, \infty}$ given by $(65) ; \boldsymbol{F}^{b}$ or $\boldsymbol{F}^{\mathrm{FL}}$ are known as multi-static response matrices. Cost functionals (16) and (17) are then accordingly replaced by appropriate finite sums. Defining the vector $\Phi_{z}^{\infty} \in \mathbb{C}^{N}$ by $\left(\Phi_{z}^{\infty}\right)_{n}=\Phi_{z}^{\infty}\left(\theta_{n}\right)=h\left(z,-\theta_{n}\right)$, the counterparts of (60) and (61) are

$$
\mathcal{T}_{\mathbb{S}}^{b}(z)=-|\mathcal{D}| k^{2} q_{\star}\left(\boldsymbol{H} \boldsymbol{\Phi}_{z}^{\infty}\right)^{\mathrm{T}} \boldsymbol{T}_{b} \overline{\boldsymbol{H} \boldsymbol{\Phi}_{z}^{\infty}}, \quad \mathcal{T}^{b}\left[\boldsymbol{\Psi}_{m}\right](z)=-|\mathcal{D}| k^{2} q_{\star} \lambda_{m}\left|\boldsymbol{\Psi}_{m}^{\mathrm{T}} \overline{\boldsymbol{\Phi}_{z}^{\infty}}\right|^{2}
$$

(with $\lambda_{m}=k^{2} Q_{m}\left\|\boldsymbol{\Phi}_{y_{m}}^{\infty}\right\|^{2} \in \mathbb{R}$ and $\boldsymbol{\Psi}_{m}=\left\|\boldsymbol{\Phi}_{y_{m}}^{\infty}\right\|^{-1} \boldsymbol{\Phi}_{y_{m}}^{\infty}$ ) while the counterpart of (67) is

$$
\mathcal{T}_{\mathbb{S}}^{\mathrm{FL}}(z)=-|\mathcal{D}| k^{2} q_{\star} \operatorname{Re}\left[\left(\boldsymbol{H} \boldsymbol{\Phi}_{z}^{\infty}\right)^{\mathrm{T}} \overline{\boldsymbol{T}^{\mathrm{FL}} \boldsymbol{H} \boldsymbol{\Phi}_{z}^{\infty}}\right] .
$$

Conclusions similar to those reached in Sections 4.2 and 4.3 hold, including Proposition 8, except for the fact that the rate of decay of $\boldsymbol{H} \boldsymbol{\Phi}_{z}^{\infty}$ as $\operatorname{dist}\left(z, D_{M}\right)$ is not known in general (i.e. for arbitrary finite sets of directions $\theta_{n}$ ); it is expected to be slower than $\operatorname{dist}\left(z, D_{M}\right)^{-1}$ in general, and to decrease with $N$.

\subsection{Numerical examples in $\mathbb{R}^{2}$}

In this section, numerical results concerning the identification of point-like scatterers in $\mathbb{R}^{2}$ are presented. The forward solution consists of the multi-static response matrix $\boldsymbol{F}^{\mathrm{FL}}$ associated with the Foldy-Lax model (see Sec. 4.4). A collection of $M=7$ point obstacles, with randomly chosen locations $y_{m} \in \mathbb{R}^{2}$ and reflectivities $Q_{m} \in \mathbb{R}$ (the latter satisfying the constraint $Q_{m} \in\left[-1+10^{-3}, 1-10^{-3}\right]$ ), is illuminated using $N=60$ incident plane waves with wave number $k=2$ and incidence directions $\theta_{n}$ equally spaced on the unit circle $\mathbb{S}$. The indicator function $\mathcal{T}_{\mathbb{S}}^{\mathrm{FL}}$ defined by (69) is then plotted, after rescaling according to (53), over the sampling region $z \in[-10 ; 10] \times[-10 ; 10]$.

Results on two such distributions of scatterers, indicated by small dots colored according to a scale indicating the value of their contrast $Q_{m}$, are presented in 


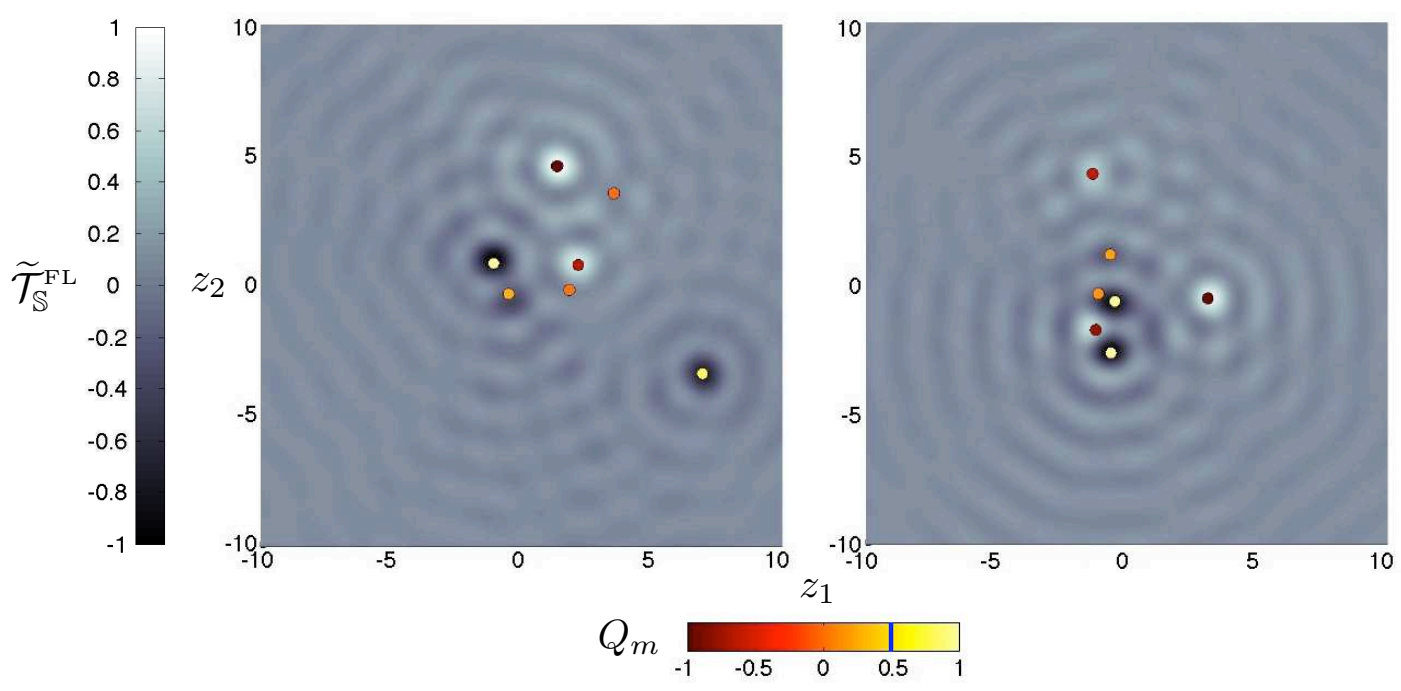

Figure 8. Identification of point-like obstacles using $\mathcal{T}_{\mathbb{S}}^{\mathrm{FL}}\left(\right.$ with $\left.q_{\star}=0.5\right)$

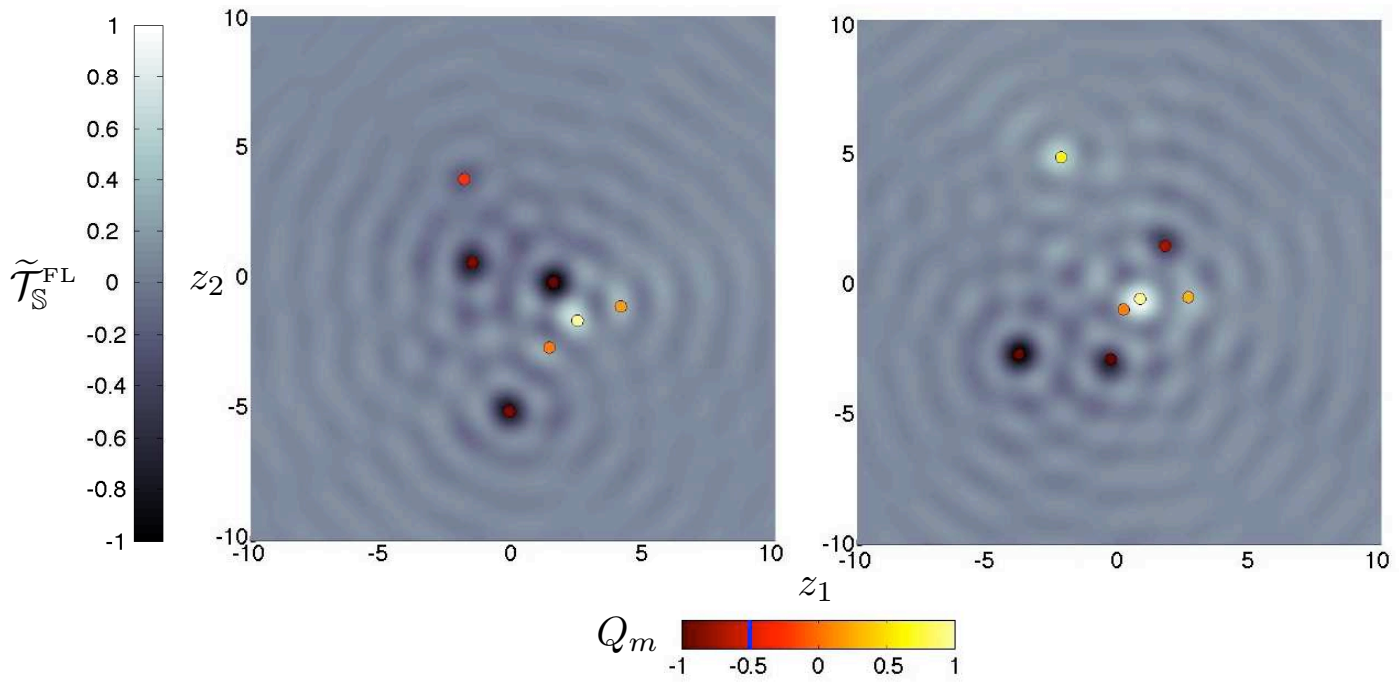

Figure 9. Identification of point-like obstacles using $\mathcal{T}_{\mathbb{S}}^{\mathrm{FL}}\left(\right.$ with $\left.q_{\star}=-0.5\right)$

Figures 8 and 9 . The two figures differ by the choice of the contrast $q_{\star}$ of the probing inhomogeneity, which was set to $q_{\star}=0.5$ for Figure 8 and to $q_{\star}=-0.5$ for Figure 9 . The function $\mathcal{T}_{\mathbb{S}}^{\mathrm{FL}}$ reaches extremal values at the locations of the scatterers having largest absolute reflectivities $\left|Q_{m}\right|$, with the corresponding extrema being negative (resp. positive) at those locations where $q_{\star} Q_{m}>0$ (resp. $\left.q_{\star} Q_{m}<0\right)$ in accordance with the sign heuristic of the method.

\section{Discussion}

\subsection{Far-field vs near-field settings}

The chosen far-field configuration plays an important role in the results of this article. In this context, the incident plane wave $h(z, \cdot)$ and the far-field pattern $\Phi_{z}^{\infty}=\overline{h(z, \cdot)}$ of 
$\Phi(z, \cdot)$ are, remarkably, mutually conjugated, leading to expression $(26 \mathrm{~b})$ of $\mathcal{T}_{\mathbb{S}}$ where $\Phi_{z}^{\infty}$ appears on both sides of the $L^{2}(\mathbb{S})$ inner product. This in turn implies that $\mathcal{T}_{\mathbb{S}}$ is expressed in terms of a weighted sum of the squared moduli of the projections of $\Phi_{z}^{\infty}$ onto the eigenfunctions of $F$, see (45). In contrast, the near-field asymptotics (20) of $v_{\varepsilon, z}$ involves the fundamental solution $\Phi(\cdot, z)$, which has no particular relationship with an incident plane wave $u_{i}$. To extend the above-described symmetry in the formulation of $\mathcal{T}(z)$ to near-field cases, one has to consider illumination by point sources, rather than plane waves, since the incident field is then also expressed in terms of $\Phi$.

\subsection{Format of the cost functional}

This study has concentrated on the topological derivative of cost functionals of leastsquares type. The concept of topological derivative is however not restricted to this particular choice. Indeed, the concept of topological derivative originates from topological optimization, where numerous formats of objective functions are used. Considering for instance a generalization of the cost functional (15) where the misfit between the trial far-field $v_{\star}^{\infty}$ and its measured value $v^{\infty}$ is evaluated using a distance function $\varphi$, the cost functional is now defined as

$$
\mathcal{J}\left(D_{\star}, q_{\star}\right):=\int_{\mathbb{S}} \varphi\left(v_{\star}^{\infty}(\hat{x})-v^{\infty}(\hat{x})\right) \mathrm{d} S_{\hat{x}} .
$$

Then advantage can be taken of an adjoint field-based formulation as it allows a generic closed-form expression of the corresponding topological derivative (see e.g. [31, 30]). Indeed, the asymptotic equality (19) associated with $\mathcal{J}$ defined by (70) takes the form

$$
\eta(\varepsilon) \mathcal{T}(z) \underset{\varepsilon \rightarrow 0}{\sim} \operatorname{Re}\left[\int_{\mathbb{S}} \varphi^{\prime}\left(-v^{\infty}(\hat{x})\right) v_{\varepsilon, z}^{\infty}(\hat{x}) \mathrm{d} S_{\hat{x}}\right],
$$

(where the derivative $\varphi^{\prime}$ of the real-valued density $\varphi(z)=\tilde{\varphi}(\operatorname{Re}(z), \operatorname{Im}(z))$ is defined as $\left.\varphi^{\prime}(z)=\left[\partial_{1}-\mathrm{i} \partial_{2}\right] \tilde{\varphi}(\operatorname{Re}(z), \operatorname{Im}(z))\right)$. Invoking the asymptotics (21) and defining the adjoint field $\hat{u}$ by

$$
\hat{u}(z):=\int_{\mathbb{S}} \varphi^{\prime}\left(-v^{\infty}(\hat{x})\right) h(z,-\hat{x}) \mathrm{d} S_{\hat{x}} .
$$

( $\hat{u}$ hence being a solution of the Helmholtz equation in $\mathbb{R}^{d}$ ), the topological derivative of (70) can finally be recast as

$$
\mathcal{T}(z)=|\mathcal{D}| k^{2} q_{\star} \operatorname{Re}\left[\hat{u}(z) u_{i}(z)\right]
$$

Applying this approach to generalizations of cost functionals $\mathcal{J}[g]$ and $\mathcal{J}_{\mathcal{S}}$ obtained by replacing $|\cdot|^{2}$ with $\varphi(\cdot)$ in (16) and (17), one similarly obtains

$$
\mathcal{T}_{\mathbb{S}}(z)=|\mathcal{D}| k^{2} q_{\star} \operatorname{Re}\left[\int_{\mathbb{S}} \hat{u}(z, \theta) h(z, \theta) \mathrm{d} S_{\theta}\right], \quad \mathcal{T}[g](z)=|\mathcal{D}| k^{2} q_{\star} \operatorname{Re}[\hat{u}(z) H g(z)]
$$

where $\hat{u}$ is defined by $(71)$ with $v^{\infty}(\hat{x})$ respectively replaced by $H g(\hat{x})$ and $A(\hat{x}, \theta)$. 
Expression (72) thus represents the generic formulation of the topological derivative of a cost functional of the form (70). Its usefulness comes from the fact that the information about the experiment, i.e. the measurements themselves and the format of the misfit function, are encapsulated in the definition of the adjoint field. It also helps in conferring flexibility to the concept of topological derivative in terms of (i) the nature and quantity of available measurements exploitable, and (ii) the available choices of cost functionals. In practice, numerical experiments on other, more complex, problems [27] indicate that the number of sources and observations can be substantially reduced while inducing only moderate degradations on the reconstructions.

\subsection{Validity of the Born approximation}

Since the most comprehensive justification of the topological derivative for scatterer identification was obtained under Born approximation conditions (Secs. 3.3 and 4.2), it is important to specify its domain of validity, which is dictated by the requirement $\left\|S T_{b}\right\| \ll 1$ (with $S$ and $T_{b}$ as defined in Sec. 2). This issue is discussed in e.g. Sec. 8.10.1 of [53], where $\left\|S T_{b}\right\| \ll 1$ is translated into the following conditions on $k, q,|D|$ using dimensional analysis (with $|D|$ denoting the $d$-dimensional volume of $D \subset \mathbb{R}^{d}$ ):

$$
k^{2}|D|^{\frac{2}{d}} \max _{D}|q| \ll 1
$$

in the low-frequency, long-wavelength limit (i.e. if $k|D|^{1 / d} \ll 1$ ), and

$$
k|D|^{\frac{1}{d}} \max _{D}|q| \ll 1
$$

in the high-frequency, short-wavelength limit (i.e. if $k|D|^{1 / d} \gg 1$ ). The numerical results of Section 3.5 are consistent with the above considerations. Since $k=10$ (Fig. 1) or $k=100$ (Figs. 2 and 3 ) and $|D|=4 \pi / 3$, all three cases can be considered as shortwavelength situations. Given the respective values of $q$ used, the Born approximation is reasonable in the first case, but not in the other two, as materialized in Fig. 4.

\subsection{Relationships with other qualitative sampling methods}

In this section, the commonalities of the topological derivative approach with some of the qualitative sampling methods among the most prominent examples mentioned in Section 1 are discussed. The far-field operator (or its discrete counterpart, the multistatic response matrix), synthesize the measurements and thus the available information on the unknown scattering object(s) that are accessible in a given excitation/observation setting. The central questions thus concern the extraction from $F$ of these informations, i.e. the reconstruction of the geometry $D$ of the obstacle and the characterization of its material contrast $q$. The so-called sampling methods [2] for inverse scattering are based on the construction of indicator functions that depend on a sampling point $z$ covering a domain of interest in $\mathbb{R}^{d}$, and which aim at providing only qualitative informations on the scatterer(s) location and material parameters, but in 
a computationally efficient framework. These techniques depart from customary, and costlier, iterative minimization approaches, which aim at quantitative reconstructions. For an overall discussion about the specific features of the topological derivative approach reference can be made to [27].

Time reversal and DORT. As discussed in [28], the topological derivative in the time-domain involves time reversal in that the adjoint solution is defined in terms of an excitation that involves time-reversed measurement residuals. For the same reason, the frequency-domain topological derivatives (24) or (25) involve the conjugated counterpart of the scattered field measurements.

Moreover, a more precise connection can be made between the topological derivative approach and the DORT method [20]. The latter aims at identifying $M$ point-like scatterers by exploiting the eigensystem of the time-reversal operator $F^{\star} F$, which is known to be given (since $F$ is normal) by $\left(\left|\lambda_{\ell}\right|^{2}, \Psi_{\ell}\right)_{\ell \in \mathbb{N}}$ (conventionally numbered so that $\left.\left|\lambda_{1}\right| \geq\left|\lambda_{2}\right| \geq \ldots\right)$ in terms of the eigensystem $\left(\lambda_{\ell}, \Psi_{\ell}\right)_{\ell \in \mathbb{N}}$ of $F$. More precisely, $\lambda_{1}, \ldots, \lambda_{M}$ are the only nonzero eigenvalues, and the incident field $u_{i}:=H \Psi_{m}$ peaks at $y_{m}$, i.e. focuses on the $m$-th scatterer (see $[43,44]$ for a mathematical justification).

The topological derivative $\mathcal{T}\left[\Psi_{\ell}\right]$ associated with the same incident fields $u_{i}:=H \Psi_{\ell}$ is in fact found to have similar focusing properties, for point-like as well as extended scatterers, and whether or not the Born approximation is used. Indeed, the magnitude of $\mathcal{T}\left[\Psi_{\ell}\right](z)$, given by (28b), (46), (61) according to the situation, consistently exhibits a $\mathcal{O}\left(\operatorname{dist}(z, D)^{1-d}\right)$ decay away from $D$. This decay, observed for a single, selective probing wave, is (i) identical to that experienced by the topological derivative $\left|\mathcal{T}_{\mathbb{S}}(z)\right|$ combining all possible directions of probing incidence, and (ii) sharper to that of $|\mathcal{T}[\theta](z)|$ corresponding to illumination by a single (or finitely many) plane waves (see Remark 5).

MUSIC. The MUSIC algorithm has been originally introduced in inverse scattering problems to detect point-like scatterers satisfying the Born approximation (i.e. within the setting of Sec. 4.2). It is based on the characterization

$$
z \in\left\{y_{1}, \ldots, y_{M}\right\} \quad \Longleftrightarrow \quad \Phi_{z}^{\infty} \in \mathcal{R}\left(H^{\star}\right)
$$

which, using that $\mathcal{R}\left(F^{0} F^{0 \star}\right)=\mathcal{R}\left(F^{0}\right)=\mathcal{R}\left(H^{\star}\right)$, leads to computing

$$
\mathcal{I}_{\text {MUSIC }}(z):=1 /\left\|P_{\mathcal{N}} \Phi_{z}^{\infty}\right\|
$$

(with the projection $P_{\mathcal{N}}=I-P$ onto the noise subspace defined in terms of the projection $P$ onto $\left.\mathcal{R}\left(F^{0} F^{0 \star}\right)\right)$ and finding the locations $z=y_{1}, \ldots, y_{M}$ at which $\mathcal{I}_{\text {MUSIC }}(z)$ has peaks.

The projection $P \Phi_{z}^{\infty}$ is found by means of a straightforward finite-dimensional least-squares minimization of $\left\|\Phi_{z}^{\infty}-H^{\star} \beta\right\|_{L^{2}(\mathbb{S})}^{2}$ with respect to $\beta \in \mathbb{C}^{M}$ (with $H$ defined by (58)) to be given by

$$
P \Phi_{z}^{\infty}(\hat{x})=\left[H^{\star} G^{-1} H \Phi_{z}^{\infty}\right](\hat{x}) \quad(\hat{x} \in \mathbb{S}),
$$

with

$$
G \in \mathbb{R}^{M \times M}, \quad G_{m n}=\int_{\mathbb{S}} h\left(y_{m}, \theta\right) \overline{h\left(y_{n}, \theta\right)} \mathrm{d} S_{\theta}=\zeta_{0}^{2}\left(y_{m}-y_{n}\right) .
$$


Noting that $H \Phi_{z}^{\infty}=\left\{\zeta_{0}\left(y_{1}-z\right), \ldots, \zeta_{0}\left(y_{M}-z\right)\right\}^{\mathrm{T}} \in \mathbb{R}^{M}$, the above result implies that $\left\|P \Phi_{z}^{\infty}\right\|_{L^{2}(\mathbb{S})}^{2}=\left(H \Phi_{z}^{\infty}\right)^{\mathrm{T}} G^{-1} H \Phi_{z}^{\infty}$. For well-separated obstacles, i.e. $k a \gg 1$ with $a$ as defined in Sec. 4.2, one has $G=4 \pi I+\mathcal{O}\left((k a)^{-2}\right)$, implying that $\left\|P \Phi_{z}^{\infty}\right\|_{L^{2}(\mathbb{S})}^{2}=$ $(4 \pi)^{-1}\left|H \Phi_{z}^{\infty}\right|^{2}+\mathcal{O}\left((k a)^{-2}\right)$, i.e. that $\left\|P \Phi_{z}^{\infty}\right\|_{L^{2}(\mathbb{S})}$ is approximately given by the 2-norm of $H \Phi_{z}^{\infty}$. One moreover observes that the topological derivative $\mathcal{T}_{\mathbb{S}}(z)$ for the same situation is given (up to a sign change and a multiplicative constant) by the weighted 2-norm $\left|H \Phi_{z}^{\infty}\right|_{\boldsymbol{T}_{b}}$ of the same vector, see (60).

Comparing $\mathcal{T}_{\mathbb{S}}(z)$ and $\mathcal{I}_{\text {MUSIC }}(z)$, the former is thus seen to exploit (a distorted version of) the projection of $\Phi_{z}^{\infty}$ onto the so-called signal subspace $\mathcal{R}(F)$, whereas the latter is based on the reciprocal of the projection of $\Phi_{z}^{\infty}$ onto the noise subspace.

Linear sampling and factorization methods. The indicator functions $\mathcal{I}_{\mathrm{LSM}}(z)$ (for the linear sampling method), $\mathcal{I}_{\mathrm{FM}}(z)$ (for the factorization method) and $\mathcal{T}_{\mathbb{S}}$ are respectively given, in terms of the orthonormal system $\left(\lambda_{\ell}, \Psi_{\ell}\right)_{\ell \in \mathbb{N}}$ of $F$ (see (44)), by

$$
\begin{aligned}
\mathcal{I}_{\mathrm{LSM}}(z) & =\left[\sum_{\ell \in \mathbb{N}} \frac{\left|\lambda_{\ell}\right|}{\left|\lambda_{\ell}\right|^{2}+\epsilon}\left|\left(\Phi_{z}^{\infty}, \Psi_{\ell}\right)_{L^{2}(\mathbb{S})}\right|^{2}\right]^{-1}, \\
\mathcal{I}_{\mathrm{FM}}(z) & =\left[\sum_{\ell \in \mathbb{N}} \frac{1}{\left|\lambda_{\ell}\right|}\left|\left(\Phi_{z}^{\infty}, \Psi_{\ell}\right)_{L^{2}(\mathbb{S})}\right|^{2}\right]^{-1}, \\
\mathcal{T}_{\mathbb{S}}(z) & =-|\mathcal{D}| k^{2} q_{\star} \sum_{\ell \in \mathbb{N}} \operatorname{Re}\left[\lambda_{\ell}\right]\left|\left(\Phi_{z}^{\infty}, \Psi_{\ell}\right)_{L^{2}(\mathbb{S})}\right|^{2}
\end{aligned}
$$

(with (45) repeated for convenience), where $\epsilon$ in $\mathcal{I}_{\mathrm{LSM}}(z)$ is a Tikhonov regularization parameter used in approximately solving for $g_{z}$ the equation $F g_{z}=\Phi_{z}^{\infty}$ (which is illposed since $F$ is compact), while $\mathcal{I}_{\mathrm{FM}}(z)$ expresses that $\Phi_{z}^{\infty} \in \mathcal{R}\left(\left(F^{\star} F\right)^{1 / 4}\right)$. All three approaches exploit the eigenvectors spanning the range of the far-field operator $F$, using the Green's function $\Phi_{z}^{\infty}$ as an available test function.

An issue of practical importance concerns the effect of measurement noise or background fluctuations on the available data $F[27,29]$. The perturbation induced by imperfect data to the evaluation of $\mathcal{T}_{\mathbb{S}}$ is linear in the data noise for the least-squares cost functional (15), and is more generally confined to the perturbation undergone by the adjoint solution $\hat{u}$ in expression (72), which is bilinear in $\left(u_{i}, \hat{u}\right)$. On the other hand, both $\mathcal{I}_{\mathrm{LSM}}$ and $\mathcal{I}_{\mathrm{FM}}$ involve the reciprocals of the eigenvalues $\lambda_{\ell}$, which makes their evaluation potentially sensitive to inaccuracies in the smallest eigenvalues. Moreover, the computation of $\mathcal{I}_{\mathrm{LSM}}(z)$ requires solving an ill-posed equation. Hence the evaluation of $\mathcal{I}_{\mathrm{LSM}}(z)$ or $\mathcal{I}_{\mathrm{FM}}(z)$ is expected to be more sensitive to noise in $F$ than that of $\mathcal{T}_{\mathbb{S}}(z)$.

Orthogonality sampling method. Owing to the relation (45), the topological derivative is conceptually comparable to the indicator function arising from the orthogonality sampling approach. The latter, recently introduced in [54] and discussed in [55], has been found to perform satisfactorily; its full mathematical justification is still open. No further insight into the topological derivative approach has so far been gained from this apparent analogy. 


\section{Conclusion}

In this article, the analysis of the topological derivative approach of inverse scattering problems by inhomogeneous acoustic media has been conducted to assess the reconstruction provided by the topological derivatives of $L^{2}$ cost functionals quantifying the misfit between measured and predicted far-field patterns. The particular structure of such misfit functions lead to imaging functionals in a form remarkably tractable in terms of analysis and comparison with other well-established qualitative and sampling methods. The sign heuristic of the method has been justified under either the Born approximation (i.e. extended inhomogeneities with weak contrast or well-separated point-like scatterers) or full-scattering models limited to moderately strong scatterers. While there is probably scope for enlarging the class of "permitted" scatterers through a more-refined analysis, a justification of the heuristic reasoning underpinning the application of the topological derivative is not expected to be achievable for arbitrarily strong scatterers. Moreover, in view of numerical evidence in some strong-scatterer regimes, e.g. high-frequency configurations where the topological derivative is observed to highlight the obstacle boundary, there may be a need to define and justify another heuristic or interpretation suitable for such situations.

If the analysis that has been carried out in this article applies to this, restricting yet widely-used, definition of the cost functional, this formulation has enabled to shed a new light on the mathematical foundations of the topological derivative approach of inverse scattering problems. One notes that the study [29] is also conducted for a least-squares measurement misfit functional.

This study represents a step towards establishing a mathematical basis supporting the topological derivative for inverse scattering and understanding its links with other

sampling approaches. Extensions of this work will address other types of inverse scattering problems, e.g. involving mass density contrasts, and the case of near-field measurements.

\section{References}

[1] A. Kirsch. An introduction to the mathematical theory of inverse problems. Springer, 2nd edition, 2011.

[2] F. Cakoni and D. Colton. Qualitative methods in inverse scattering theory. Springer-Verlag, Berlin, 2006.

[3] R. Potthast. A survey on sampling and probe methods for inverse problems. Inverse Problems, 22:R1-R47, 2006.

[4] P. Lax and R. S. Phillips. Scattering theory. Academic Press, 1967.

[5] D. Colton and R. Kress. Inverse acoustic and electromagnetic scattering theory. Springer, Berlin, 1992.

[6] D. Colton and R. Kress. Eigenvalues of the far field operator and inverse scattering theory. SIAM J. Math. Anal., 26:601-615, 1995.

[7] A. Kirsch. Characterization of the shape of a scattering obstacle using the spectral data of the far field operator. Inverse Problems, 14:1489-1512, 1998. 
[8] T. D. Mast, A. I. Nachman, and R. C. Waag. Focusing and imaging using eigenfunctions of the scattering operator. J. Acoust. Soc. Am., 102:715-725, 1997.

[9] T. Arens and A Lechleiter. The linear sampling method revisited. J. Integral Equations Appl., 21:179-202, 2009.

[10] A. Kirsch and N. Grinberg. The Factorization Method for Inverse Problems. Oxford University Press, New York, 2008.

[11] M. Cheney. The linear sampling method and the MUSIC algorithm. Inverse Problems, 17:591-595, 2001.

[12] A. Kirsch. The MUSIC-algorithm and the factorization method in inverse scattering theory for inhomogeneous media. Inverse Problems, 18:1025-1040, 2002.

[13] A. J. Devaney. Super-resolution processing of multi-static data using time reversal and MUSIC. http://www.ece.neu.edu/faculty/devaney/preprints/paper02n_00.pdf , Northeastern University Preprint, 2000,

[14] H. Ammari and H. Kang. Reconstruction of small inhomogeneities from boundary measurements. Lecture Notes in Mathematics 1846. Springer-Verlag, 2004.

[15] A. J. Devaney. Time reversal imaging of obscured targets from multistatic data. IEEE Trans. Antennas Propag., 53:1600-1610, 2005.

[16] D. Cassereau, F. Wu, and M. Fink. Limits of self-focusing using closed time-reversal cavities and mirrors - theory and experiment. IEEE Ultrasonics Symposium, 3:1613-1618, 1990.

[17] M. Fink. Time reversal of ultrasonic fields - part I: Basic principles. IEEE Trans. Ultrason., Ferroelec., Freq. Contr., 39:555-566, 1992.

[18] C. Prada, J. L. Thomas, and M. Fink. The iterative time reversal process: Analysis of the convergence. J. Acoust. Soc. Am., 97:62-71, 1995.

[19] L. Borcea, G. C. Papanicolaou, C. Tsogka, and J. G. Berrymann. Imaging and time reversal in random media. Inverse Problems, 18:1247-1279, 2002.

[20] C. Prada and M. Fink. Eigenmodes of the time reversal operator: A solution to selective focusing in multiple target media. Wave motion, 20:151-163, 1994.

[21] C. Prada, S. Manneville, D. Spoliansky, and M. Fink. Decomposition of the time reversal operator: Application to detection and selective focusing on two scatterers. J. Acoust. Soc. Am., 99:20672076, 1996.

[22] C. Prada, M. Tanter, and M. Fink. Flaw detection in solid with the D.O.R.T. method. IEEE Ultrason. Symp. Proc., 1:679-683, 1997.

[23] H. A. Eschenauer, V. V. Kobelev, and A. Schumacher. Bubble method for topology and shape optimization of structures. Structural Optimization, 8:42-51, 1994.

[24] L. Jackowska-Strumillo, J. Sokolowski, and A. Zochowski. Topological optimization and inverse problems. Computer Assisted Mechanics and Engineering Sciences, 10(2):163-176, 2003.

[25] M. Bonnet and B. B. Guzina. Sounding of finite solid bodies by way of topological derivative. Int. J. Num. Meth. in Eng., 61:2344-2373, 2004.

[26] B. B. Guzina and I. Chikichev. From imaging to material identification: a generalized concept of topological sensitivity. J. Mech. Phys. Solids, 55:245-279, 2007.

[27] C. Bellis and M. Bonnet. A FEM-based topological sensitivity approach for fast qualitative identification of buried cavities from elastodynamic overdetermined boundary data. Int. J. Solids Struct., 47:1221-1242, 2010.

[28] N. Dominguez, V. Gibiat, and Y. Esquerré. Time domain topological gradient and time reversal analogy: an inverse method for ultrasonic target detection. Wave Motion, 42:31-52, 2005.

[29] H. Ammari, J. Garnier, V. Jugnon, and H. Kang. Stability and resolution analysis for a topological derivative based imaging functional. SIAM J. Control Optim., 50:48-76, 2012.

[30] B. B. Guzina and M. Bonnet. Small-inclusion asymptotic of misfit functionals for inverse problems in acoustics. Inverse Problems, 22:1761-1785, 2006.

[31] M. Bonnet. Inverse acoustic scattering by small-obstacle expansion of a misfit function. Inverse Problems, 24:035022, 2008. 
[32] A. Laurain, M. Hintermüller, M. Freiberger, and H. Scharfetter. Topological sensitivity analysis in fluorescence optical tomography. Inverse Problems, 29:025003, 2013.

[33] F. W. J. Olver, D. W. Lozier, R. F. Boisvert, and C. W. Clark, editors. NIST handbook of mathematical functions. Cambridge, 2010.

[34] H. Ammari, E. Iakovleva, and S. Moskow. Recovery of small inhomogeneities from the scattering amplitude at a fixed frequency. SIAM J. Math. Anal., 34:882-890, 2003.

[35] E. M. Stein. Harmonic analysis: real-variable methods, orthogonality, and oscillatory integrals. Princeton University Press, 1993.

[36] E. M. Stein and G. Weiss. Introduction to Fourier analysis on Euclidean spaces. Princeton University Press, 1971.

[37] A. Erdélyi, editor. Tables of Integral Transforms, volume II, Bateman Manuscript Project. McGraw-Hill Book Co., New York, 1954.

[38] D. Colton and R. Kress. Eigenvalues of the far field operator for the helmholtz equation in an absorbing medium. SIAM J. Appl. Math., 55:1724-1735, 1995.

[39] K. Kilgore, S. Moskow, and J. C. Schotland. Inverse Born series for scalar waves. J. Comput. Math., 30:601-614, 2012.

[40] B. B. Guzina, F. Cakoni, and C. Bellis. On the multi-frequency obstacle reconstruction via the linear sampling method. Inverse Problems, 26:125005, 2010.

[41] G. Vainikko. Fast solvers of the Lippmann-Schwinger equation. In Direct and inverse problems of mathematical physics, R. P. Gilbert, J. Kajiwara, Y. S. Xu (eds), pp. 423-440, Kluwer Academic Publishers, 2000.

[42] C. Hazard and K. Ramdani. Selective acoustic focusing using time-harmonic reversal mirrors. SIAM J. Appl. Math., 64:1057-1076, 2004.

[43] B. Pinçon and K. Ramdani. Selective focusing on small scatterers in acoustic waveguides using time reversal mirrors. Inverse Problems, 23:1-25, 2007.

[44] C. Burkard, A. Minut, and K. Ramdani. Far-field model for time reversal and application to selective focusing on small dielectric inhomogeneities. Inverse Problems in Imaging, 7:445-470, 2013.

[45] M. Brühl, M. Hanke, and M. S. Vogelius. A direct impedance tomography algorithm for locating small inhomogeneities. Numer. Math., 93:635-654, 2003.

[46] H. Ammari, E. Iakovleva, D. Lesselier, and G. Perrusson. MUSIC-type electromagnetic imaging of a collection of small three-dimensional inclusions. SIAM J. Sci. Comput., 29:674-709, 2007.

[47] E. Iakovleva, S. Gdoura, and D. Lesselier. Multi-static response matrix of a 3-d inclusion in a half space and MUSIC imaging. IEEE Trans. Antennas Propag., 55:2598-2609, 2007.

[48] H. Ammari, J. Garnier, and K. Sølna. A statistical approach to optimal target detection and localization in the presence of noise. Waves in Random and Complex Media, 22:40-65, 2012.

[49] L. L. Foldy. The multiple scattering of waves. Phys. Rev., 67:107-119, 1945.

[50] M. Lax. Multiple scattering of waves. Rev. Mod. Phys., 23:287-310, 1951.

[51] A. J. Devaney, E. A. Marengo, and F. K. Gruber. Time-reversal-based imaging and inverse scattering of multiply scattering point targets. J. Acoust. Soc. Am., 118:3129-3138, 2005.

[52] D. P. Challa and M. Sini. Inverse scattering by point-like scatterers in the foldy regime. Inverse Problems, 28:125006, 2012.

[53] W. C. Chew. Waves and fields in inhomogeneous media. IEEE Press, New York, 1995.

[54] R. Potthast. A study on orthogonality sampling. Inverse Problems, 26:074015, 2010.

[55] R. Griesmaier. Multi-frequency orthogonality sampling for inverse obstacle scattering problems. Inverse Problems, 27:085005, 2011. 